\title{
Randomized approximate nearest neighbor search with limited adaptivity
}

\author{
Mingmou Liu ${ }^{*} \quad$ Xiaoyin Pan ${ }^{\dagger} \quad$ Yitong Yin ${ }^{\ddagger}$
}

\begin{abstract}
We study the fundamental problem of approximate nearest neighbor search in $d$-dimensional Hamming space $\{0,1\}^{d}$. We study the complexity of the problem in the famous cell-probe model, a classic model for data structures. We consider algorithms in the cell-probe model with limited adaptivity, where the algorithm makes $k$ rounds of parallel accesses to the data structure for a given $k$. For any $k \geq 1$, we give a simple randomized algorithm solving the approximate nearest neighbor search using $k$ rounds of parallel memory accesses, with $O\left(k(\log d)^{1 / k}\right)$ accesses in total. We also give a more sophisticated randomized algorithm using $O\left(k+\left(\frac{1}{k} \log d\right)^{O(1 / k)}\right)$ memory accesses in $k$ rounds for large enough $k$. Both algorithms use data structures of size polynomial in $n$, the number of points in the database.

For the lower bound, we prove an $\Omega\left(\frac{1}{k}(\log d)^{1 / k}\right)$ lower bound for the total number of memory accesses required by any randomized algorithm solving the approximate nearest neighbor search within $k \leq \frac{\log \log d}{2 \log \log \log d}$ rounds of parallel memory accesses on any data structures of polynomial size. This lower bound shows that our first algorithm is asymptotically optimal for any constant round $k$. And our second algorithm approaches the asymptotically optimal tradeoff between rounds and memory accesses, in a sense that the lower bound of memory accesses for any $k_{1}$ rounds can be matched by the algorithm within $k_{2}=O\left(k_{1}\right)$ rounds. In the extreme, for some large enough $k=\Theta\left(\frac{\log \log d}{\log \log \log d}\right)$, our second algorithm matches the $\Theta\left(\frac{\log \log d}{\log \log \log d}\right)$ tight bound for fully adaptive algorithms for approximate nearest neighbor search due to Chakrabarti and Regev [10].
\end{abstract}

\section{Introduction}

Nearest neighbor search is a fundamental theoretical problem in Computer Science, with enormously many applications in diverse fields. In the nearest neighbor search problem, we are given a database $B$ of $n$ points from a metric space $X$. The goal is to preprocess them into a data structure, such that given any query point $x \in X$, an algorithm with accessing to the data structure can find a database point in $B$ that is closest to the query point $x$ among all database points. An extensively studied case is when the metric space is the Hamming space $X=\{0,1\}^{d}$.

It is conjectured that the nearest neighbor search is hard to solve by any data structures when the dimension $d$ is high (e.g. $d \gg \log n$ ). This conjecture is sometimes referred as a case of the "curse of dimensionality" and is one of the central problems in the area of data structure lower

\footnotetext{
${ }^{*}$ Department of Computer Science and Technology, Nanjing University, China. MG1533028@smail.nju.edu.cn

${ }^{\dagger}$ Department of Computer Science and Technology, Nanjing University, China. xiaoyin.pan95@gmail.com

${ }^{\ddagger}$ State Key Laboratory for Novel Software Technology, Nanjing University, China. Supported by NSFC grants no. 61272081 and 61321491. Email: yinyt@nju.edu.cn.
} 
bounds. It is also believed that the problem of high-dimensional nearest neighbor search remains to be intractable while either an approximation is tolerated or the algorithm is randomized, but not both at the same time [1].

The complexity of the nearest neighbor search problem, as well as many other data structure problems, is well studied in the cell-probe model [24, a classic model for the complexity of data structures. In the cell-probe model, the database is preprocessed into a data structure, stored as a table in the main memory, and upon each query, an algorithm, called the cell-probing algorithm, outputs an answer to the query after adaptively probing a number of table cells. The complexity is measured by both the size of the data structure and the number of cell-probes made by the algorithm to answer a query in the worst case. There is a substantial body of works on the cellprobe complexity of nearest neighbor search in Hamming space [6, 7, 13, 16, 19, 21].

When both approximation and randomization are allowed, a seminal work of Chakrabarti and Regev [10] gives a tight bound for the complexity of nearest neighbor search in $d$-dimensional Hamming space with data structures of size polynomial in $n$, assuming the dimension $d$ is high (and not too high to trivialize the problem, e.g. $(\log n)^{1.01} \leq d \leq 2^{\sqrt{\log n}}$ ). This fundamental result is stated informally as follows.

Theorem 1 (Chakrabarti and Regev [10]) Assume $(\log n)^{1.01} \leq d \leq 2^{\sqrt{\log n}}$. The cell-probe complexity of randomized approximate nearest neighbor search in d-dimensional Hamming space in the cell-probe model with data structure of polynomial size is $\Theta\left(\frac{\log \log d}{\log \log \log d}\right)$.

On the other hand, when the table size becomes closer to be linear of $n$, data structures such as locality-sensitive hashing (LSH) [2,12] or data-dependent LSH 3,4, achieve a cell-probe complexity of $\tilde{O}\left(d n^{\rho}\right)$ with data structures of size $\tilde{O}\left(n^{1+\rho}\right)$ for some $0<\rho<1$ depending on the metric and the approximation ratio. Compared to the $\Theta\left(\frac{\log \log d}{\log \log \log d}\right)$ bound of Chakrabarti and Regev, the $\tilde{O}\left(d n^{\rho}\right)$ cell-probe complexity is much worse. The popularity in practice of the LSH-based data structures is due to their low space cost, and the ability to be implemented in parallel.

Take locality-sensitive hashing (LSH) as an example. The algorithm of LSH is non-adaptive: Each cell-probe relies only on the query but not on the information retrieved by other cell-probes. This makes all cell-probes in LSH parallelizable into one round of parallel memory accesses. And the more recent data-dependent LSH [3, 4] surpasses the classic LSH in cell-probe complexity by being a little more adaptive: the algorithm retrieves a data-dependent hash function before making the second round of cell-probes, while the cell-probes in the second round are independent of each other. In contrast, the algorithm of Chakrabarti and Regev [10] is fully adaptive: Every cell-probe must wait for the information retrieved by the previous cell-probe to proceed.

This could give us the following intuitive image: A cell-probing algorithm is getting more clever and hence more efficient, as it is becoming more adaptive. It is then a fundamental question to study the tradeoff between the efficiency (measured by the total number of cell-probes) and adaptivity (measured by the number of rounds of parallel cell-probes) in the cell-probe model. Very little was known to this fundamental question. In [8], Brody and Larsen initiated the study of non-adaptive dynamic data structures, where the database receives both queries and updates. They show a foundational result that for dynamic data structures, being adaptive is critical. For static data structures, parallel cell-probes were studied in the context of low-contention data structures [5,20]. The highest cell-probe lower bound known for non-adaptive static data structure is the $\Omega\left(\log n / \log \frac{s d}{n \log n}\right)$ cell-probe lower bound of Panigrahy, Talwar, and Wieder [19] for randomized 
approximate nearest neighbor search on a table of size $s$. This lower bound becomes trivial for tables of polynomial size. For cell-probe model with polynomial-sized data structures, the tradeoff between the cell-probe complexity and adaptivity is highly unknown for any static data structure problems.

Our results. In this paper, we study the complexity of randomized approximate nearest neighbor search in the cell-probe model with limited adaptivity. We consider a natural notion of rounds for cell-probes, where the cell-probes in the same round are adaptive to the information retrieved in previous rounds, but non-adaptive to each other in the same round.

We give two randomized cell-probing algorithms for approximate nearest neighbor search in $d$-dimensional Hamming space. For both algorithms, the data structures are of polynomial size, and the cell-probes are organized into $k$ rounds for any $k \geq 1$ (Algorithm 1) or for all sufficiently large $k$ (Algorithm 2). The first algorithm is as follow.

Theorem 2 For any $k \geq 1$, the approximate nearest neighbor search in d-dimensional Hamming space can be solved in the cell-probe model with a data structure of polynomial size, using $k$ rounds of parallel randomized cell-probes, with $O\left(k(\log d)^{1 / k}\right)$ cell-probes in total.

The algorithm is simple and works for all $k \geq 1$ number of rounds. Especially when $k=1$, the algorithm is non-adaptive. Compared to the LSH which is also non-adaptive, our algorithm achieves a much better cell-probe complexity $O(\log d)$ by using a data structure of larger polynomial size.

However, when the round number $k$ becomes large, especially at the extreme when every round has 1 cell-probe, in which case the algorithm becomes fully adaptive and has $O(\log \log d)$ total cell-probes, which is not optimal for fully adaptive algorithms by Theorem 1. This leads us to our second more sophisticated algorithm.

Theorem 3 For large enough $k$, the approximate nearest neighbor search in d-dimensional Hamming space can be solved in the cell-probe model with a data structure of polynomial size, using $k$ rounds of parallel randomized cell-probes, with $O\left(k+\left(\frac{1}{k} \log d\right)^{O(1 / k)}\right)$ cell-probes in total.

The second algorithm is substantially more sophisticated. In the extreme, it approaches the optimal fully adaptive algorithm in Theorem 1 in the following sense: For some sufficiently large $k=O\left(\frac{\log \log d}{\log \log \log d}\right)$, we can implement the algorithm such that every round of the algorithm contain only 1 cell-probe.

We emphasize that these algorithms are not meant to be efficient in practice due to their expensive space costs, rather, they are parts of a theoretical endeavor to understand the complexity tradeoff between time and rounds on data structures of polynomial size. With this spirit, we prove the following lower bound for the tradeoff between cell-probe complexity and round complexity for randomized approximate nearest neighbor search.

Theorem 4 Assume $(\log n)^{1.01} \leq d \leq 2^{\sqrt{\log n}}$ and $1 \leq k \leq \frac{\log \log d}{2 \log \log \log d}$. Any randomized algorithm solving the approximate nearest neighbor search in d-dimensional Hamming space in the cell-probe model with a data structure of polynomial size using $k$ rounds of parallel randomized cell-probes must use $\Omega\left(\frac{1}{k}(\log d)^{1 / k}\right)$ cell-probes in total.

Due to this lower bound, both our algorithms achieve some optimality: 
- Algorithm 1 is asymptotically optimal in cell-probe complexity for any constant number of rounds.

- Algorithm 2 approaches the asymptotically optimal tradeoff between cell-probe complexity and round complexity in the following sense: the cell-probe lower bound for any $k_{1}$-round algorithms can be approached by Algorithm 2 within $k_{2}=O\left(k_{1}\right)$ rounds.

In addition, Algorithm 2 together with our lower bound show that the cell-probe complexity of randomized approximate nearest neighbor search undergoes a "phase transition" when the round number is within the regime $k=\Theta\left(\frac{\log \log d}{\log \log \log d}\right)$ : For a small $k_{1}=\Theta\left(\frac{\log \log d}{\log \log \log d}\right)$, the average number of cell-probes per each round for any $k_{1}$-round algorithm has to be a $(\log \log d)^{\Omega(1)}$, whereas for large enough $k_{2}=\Theta\left(\frac{\log \log d}{\log \log \log d}\right)$, only 1 cell-probe in each round is enough for a $k_{2}$-round algorithm.

Technique. Both our upper bounds and lower bounds rely heavily on the machineries developed in [10].

The main ideas for the upper bounds are the dimension reduction techniques developed in the pioneering works of [12,15] and the multi-way search in [10. Our efforts are focused on how to apply these techniques to give a family of algorithms approaching the smoothed tradeoff between round and cell-probe complexity. A technical innovation of [10] is to use two kinds of approximations of Hamming balls: an accurate approximation of hamming ball which is more expensive, and a coarse approximation which is cheap, to support a multi-way search with a substantial number of branchings, such that each branching is supported by one query to an accurate ball succeeded by several queries to coarse balls, which altogether consume only $O(1)$ cell-probes. Surprisingly, we discover that a simple algorithm can achieve an optimal cell-probe complexity in any constant number of rounds, using only the more expensive accurate approximation of Hamming balls. And for general round numbers, the coarse approximation of balls are employed to approach the asymptotically optimal tradeoff between rounds and cell-probes.

The lower bound is proved by the round elimination of communication protocols for the longest prefix matching problem LPM, which can be reduced to approximate nearest neighbor search. Usually the data structure lower bounds are proved for a decision version of the problem. For nearest neighbor search, a natural decision version is the $\lambda$-near neighbor problem $\lambda$-NN. However, it is folklore that with both approximation and randomization allowed, $\lambda$-NN can be solved within $O(1)$-probe on a table of polynomial size. So to prove a nontrivial lower bound in this case, one must stay with the search problem. In [10, this is done by a reduction from the longest prefix matching LPM, a problem that critically captures the nature of searching for the nearest neighbors. In [10], a lower bound is proved for LPM by interpreting a data structure as a communication protocol and applying round eliminations to the communication protocol, a technique that can be traced back to [1,17]. Our main observation is that $k$ rounds of cell-probes can be represented as $2 k$ rounds of communications. Although the observation is straightforward, to prove our lower bound we have to apply the techniques of [10] to adapt to non-uniform message sizes in different rounds, a setting which was rarely considered in the context of asymmetric communication complexity for data structure lower bounds. More critically, in order to get the $1 / k$ exponent in our $\Omega\left(\frac{1}{k}(\log d)^{1 / k}\right)$ lower bound, we are forced to exploit the round elimination of [10]. In fact, assuming $k=O\left(\frac{\log \log d}{\log \log \log d}\right)$, a lower bound with form $\Omega\left(k+\frac{1}{k^{b}}(\log d)^{a / k}\right)$ for any constants $a, b>0$ is enough to imply the 
optimal $\Omega\left(\frac{\log \log d}{\log \log \log d}\right)$ lower bound in Theorem 1, whereas for our result, these constants $a, b$ matter a lot and require much delicacy in the round elimination argument.

\section{Preliminaries}

Approximate nearest neighbor search: We consider the problem of approximate nearest neighbor search in the $d$-dimensional Hamming space ANNS $_{d, n}^{\gamma}$. Let $\gamma>1$ be fixed. We are given a database $B$ which contains $n$ points from the $d$-dimensional Hamming cube $\{0,1\}^{d}$. The database is preprocessed into a data structure (called the table). Then given any query point $x \in\{0,1\}^{d}$, the algorithm must access the data structure and output a database point $y \in B$ which is a $\gamma$-approximate nearest neighbor of $x$ in $B$, where a point $y \in B$ is called a $\gamma$-approximate nearest neighbor of $x$ in $B$ if $\operatorname{dist}(x, y) \leq \gamma \cdot \min _{z \in B} \operatorname{dist}(x, z)$, where $\operatorname{dist}(x, y)$ denotes the Hamming distance between $x$ and $y$.

Abstractly, a data structure problem can be represented as a relation $\rho \subseteq \mathscr{A} \times \mathscr{B} \times \mathscr{C}$, where $\mathscr{A}, \mathscr{B}$, and $\mathscr{C}$ specify the universes for queries, databases, and answers, respectively. Given a query $x \in \mathscr{A}$ to a database $B \in \mathscr{B}$, an answer $z \in \mathscr{C}$ is correct if $(x, B, z) \in \rho$. In particular, for approximate nearest neighbor search, $\mathscr{A}=\mathscr{C}=\{0,1\}^{d}, \mathscr{B}=\left(\begin{array}{c}\{0,1\}^{d} \\ n\end{array}\right)$, and

$$
\operatorname{ANNS}_{d, n}^{\gamma}=\{(x, B, z) \in \mathscr{A} \times \mathscr{B} \times \mathscr{C} \mid z \in B \wedge \forall y \in B, \operatorname{dist}(x, z) \leq \gamma \cdot \operatorname{dist}(x, y)\} .
$$

The cell-probe model. We adopt Yao's cell-probe model 24] for static data structures. A cell-probing scheme $(\mathcal{A}, \mathcal{T})$ for a data structure problem $\rho \subseteq \mathscr{A} \times \mathscr{B} \times \mathscr{C}$ consists of a cell-probing algorithm $\mathcal{A}$ and a code (sometimes called the table structure) $\mathcal{T}$. Each database $B \in \mathscr{B}$ is mapped by the code $\mathcal{T}: \mathscr{B} \rightarrow \Sigma^{s}$ to a codeword (called a table) $T_{B} \in \Sigma^{[s]}$ of $s$ symbols from the alphabet $\Sigma=\{0,1\}^{w}$. The interpretation is that each database $B$ is preprocessed and stored as a table $T_{B}$ consisting of $s$ table cells (also called a word), with each cell storing $w$ bits. Given an address $i \in[s]$ we use $T_{B}[i]$ to denote the content of the $i$-th cell in table $T_{B}$. Given a query $x \in \mathscr{A}$, the cell-probing algorithm $\mathcal{A}$ must output a correct answer $z \in \mathscr{C}$ such that $(x, B, z) \in \rho$, after accessing the table $T_{B}$ adaptively for $t$ times, each time reading the content of one table cell. This action of reading the content of one table cell by the cell-probing algorithm is usually called as making a cell-probe.

The complexity of a cell-probing scheme is captured by three parameters: namely, the table size $s$, the word size $w$, and the time cost or cell-probe complexity $t$.

Cell-probe model with limited adaptivity: In this work, we refine the cell-probe model by considering the rounds of parallelizable cell-probes in cell-probing algorithms. Formally, a $k$-round cell-probing algorithm $\mathcal{A}$ can be described by $k$ lookup functions $L_{1}, L_{2}, \ldots, L_{k}$ and one truth table $A$. Each lookup function $L_{i}$ maps the query $x$ and the contents of the table cells probed before round $i$, to a sequence of addresses indicating the set of table cells to probe in round $i$. In the beginning, $L_{1}(x)=\left(p_{1}^{1}, p_{2}^{1}, \ldots, p_{t_{1}}^{1}\right) \in[s]^{t_{1}}$ for some $t_{1}>0$, and for general $1 \leq i \leq k$ :

$$
L_{i}\left(x,\left\langle p_{\ell}^{j}, T_{B}\left[p_{\ell}^{j}\right]\right\rangle_{\substack{1 \leq j<i \\ 1 \leq \ell \leq t_{j}}}\right)=\left(p_{1}^{i}, p_{2}^{i}, \ldots, p_{t_{i}}^{i}\right) \in[s]^{t_{i}}, \quad \text { for some } t_{i}>0,
$$


so that at round $i$, the algorithm makes $t_{i}$ parallel cell-probes to the the cells $p_{\ell}^{i}, 1 \leq \ell \leq t_{i}$. And finally, the truth table $A$ maps the contents of all the probed cells $\left\langle p_{\ell}^{j}, T_{B}\left[p_{\ell}^{j}\right]\right\rangle_{\substack{1 \leq j \leq k \\ 1 \leq \ell \leq t_{j}}}$, to a correct answer $z$ satisfying that $(x, B, z) \in \rho$. The cell-probe complexity is given by $t=t_{1}+t_{2}+\cdots+t_{k}$. This formulation includes the standard definition of cell-probing scheme as a special case when $t_{1}=\cdots=t_{k}=1$.

Public-coin vs. private-coin cell-probing schemes: In a (private-coin) randomized cellprobing scheme, the table is prepared by a code $\mathcal{T}$ deterministically as before, but the cell-probing algorithm $\mathcal{A}$ is a randomized algorithm. This can be considered as that the deterministic lookup functions $L_{1}, L_{2}, \ldots, L_{k}$ as well as the truth table $A$ also take a sequence of random bits $r \in\{0,1\}^{*}$ as part of the input. We say we have a randomized cell-probing scheme $(\mathcal{A}, \mathcal{T})$ for a data structure problem $\rho \subseteq \mathscr{A} \times \mathscr{B} \times \mathscr{C}$ if for every query $x \in \mathscr{A}$ and every database $B \in \mathscr{B}$, the cell-probing algorithm outputs a correct answer $z \in \mathscr{C}$ such that $(x, B, z) \in \rho$ with probability at least $2 / 3$. The constant $2 / 3$ is rather arbitrary. Note that for problems such as approximate nearest neighbor search, where once the query $x$ is known, a monotone order of the correctness between multiple answers is fixed, any positive constant success probability is enough: we can boost it to any constant accuracy $1-\epsilon$ by independent repetition of the cell-probing algorithm for constant many times in parallel, which will keep the asymptotic cell-probe complexity and the number of rounds of parallel cell-probes.

In this paper, all of our upper bounds will be presented first as public-coin randomized cellprobing schemes. For a public-coin randomized cell-probing scheme, the sequence of random bits $r \in\{0,1\}^{*}$ is shared between the cell-probing algorithm $\mathcal{A}$ and the table structure $\mathcal{T}$, where the table $T_{B}^{r}$ is now determined by both the database $B$ and the random bits $r$. This makes no change to the family of data structures of polynomial size: by Newman's theorem [18, a public-coin cell-probing scheme can be transformed to a standard randomized cell-probing scheme, where the randomness is private to the cell-probing algorithm.

Lemma 5 If there is a $k$-round public-coin randomized cell-probing scheme for a data structure problem $\rho \subseteq \mathscr{A} \times \mathscr{B} \times \mathscr{C}$ with table size $s$, word size $w$, and cell-probe complexity $t$, then there is a $k$-round randomized cell-probing scheme for $\rho$ with table size $(\log |\mathscr{A}|+\log |\mathscr{B}|+O(1))$ s, word size $w$, and cell-probe complexity $t$.

Proof: The proof is similar to the proof of Lemma 6.5 in [10], with the observation that the translation there also preserves the number of rounds. Without loss of generality, we assume that for every query to every database, the $k$-round public-coin randomized cell-probing scheme returns a correct answer except with an error probability at most $1 / 4$. The $k$-round public-coin randomized cell-probing scheme can be seen as a $k$-round public coin randomized communication protocol between Alice for the cell-probing algorithm and Bob for the table, where Bob is nonadaptive thus is only response to each individual message received in the current round according to its input $B$ in a consistent way (as a code). By Newman's theorem, the number of public random bits can be reduced to $\ell=\log (\log |\mathscr{A}|+\log |\mathscr{B}|+O(1))$ while the error probability is raised to $1 / 3$. This does not change the structure of the protocol, so it can be translated back to a $k$-round public-coin randomized cell-probing scheme for $\rho$ with the same time and space complexity as before and with $\ell$ public random bits. We create a table $T_{B}^{r}$ for every possible sequence of random bits $r \in\{0,1\}^{\ell}$ according to the public-coin cell-probing scheme. This gives us a big table of 
size $s \cdot 2^{\ell}=s(\log |\mathscr{A}|+\log |\mathscr{B}|+O(1))$, and the random bits is made private to the cell-probing algorithm.

Notations. We use dist $(\cdot, \cdot)$ to denote Hamming distance. We write log for binary logarithm and ln for natural logarithm.

\section{Approximate nearest neighbor search in $k$ rounds}

In this section, we will give two algorithms that solve the approximate nearest neighbor search problem $\mathrm{ANNS}_{d, n}^{\gamma}$ within $k$ rounds on a table of size $n^{O(1)}$ and word size $O(d)$ :

1. a simple $k$-round randomized cell-probing scheme with $O\left(k(\log d)^{1 / k}\right)$ cell-probes;

2. a more sophisticated $k$-round randomized cell-probing scheme with $O\left(k+\left(\frac{1}{k} \log d\right)^{c / k}\right)$ cellprobes, for any constant $c>2$.

We will also include a folklore result in the current framework to show that if the problem is relaxed a little to the approximate near-neighbor search problem (instead of the nearest neighbor search), then on a table of polynomial size with word size $O(d)$, the problem can be solved with $O(1)$ cell-probes by a non-adaptive (i.e. 1-round) randomized cell-probing scheme.

Public-coin vs. private-coin in the cell-probe model. All our three algorithms will be first presented as public-coin cell-probing schemes, where the random bits are shared between the cellprobing algorithm and the table, and then transformed by Lemma 5 to the standard randomized cell-probing schemes, where the random bits are private to the cell-probing algorithm, with the same round and cell-probe complexity and a polynomial overhead to the table size. In particular, for $\mathrm{ANNS}_{d, n}^{\gamma}$ we have the following proposition.

Proposition 6 If there is a k-round public-coin randomized cell-probing scheme for ANNS $_{d, n}^{\gamma}$ with table size $s$, word size $w$, and cell-probe complexity $t$, there exists a $k$-round randomized cell-probing scheme for $\mathrm{ANNS}_{d, n}^{\gamma}$ with table size $O(d n \cdot s)$, word size $w$, and cell-probe complexity $t$.

Common setup for the algorithms. We consider only constant approximation ratio $\gamma>1$, so without lost of generality, we can assume that $\gamma<4$, since for larger $\gamma$ our algorithms will only have better approximation. Let $\alpha \triangleq \sqrt{\gamma}$, and hence $1<\alpha<2$. Let $x \in\{0,1\}^{d}$ denote the query point and $B \subseteq\{0,1\}^{d},|B|=n$, denote the database. We always assume that $n>d$. For $\left.0 \leq i \leq\left\lceil\log _{\alpha} d\right\rceil\right\}$, let

$$
B_{i}=\left\{y \in B \mid \operatorname{dist}(x, y) \leq \alpha^{i}\right\}
$$

be the set of all database points within distance $\alpha^{i}$ of $x$.

Definition 7 Let $c_{1}, c_{2}>64 /\left(1-\mathrm{e}^{(1-\alpha) / 2}\right)^{2}$ be constants and $1<s<\ln \ln n$. For $0 \leq i \leq\left\lceil\log _{\alpha} d\right\rceil$, let $M_{i}, N_{i}$ be the independent random Boolean matrices such that each entry is sampled i.i.d. from 
$\operatorname{Bernoulli}\left(\frac{1}{4 \alpha^{i}}\right)$, with $M_{i}$ of size $\left(c_{1} \log n\right) \times d$ and $N_{i}$ of size $\left(\frac{c_{2}}{s} \log n\right) \times d$. For $0 \leq j \leq i \leq\left\lceil\log _{\alpha} d\right\rceil$, we define the sets

$$
\begin{aligned}
C_{i} & =\left\{z \in B \mid \operatorname{dist}\left(M_{i} x, M_{i} z\right) \leq \delta\left(\alpha^{i}, \alpha\right) \cdot c_{1} \log n\right\}, \\
D_{i, j} & =\left\{z \in C_{i} \mid \operatorname{dist}\left(N_{j} x, N_{j} z\right) \leq \delta\left(\alpha^{j}, \alpha\right) \cdot\left(c_{2} \log n\right) / s\right\},
\end{aligned}
$$

where $\delta(\beta, \alpha)=\frac{1}{2}\left(1-\frac{1}{2 \beta}\right)^{\beta}\left[1-\left(1-\frac{1}{2 \beta}\right)^{(\alpha-1) \cdot \beta}\right]$.

The following lemma proved in [10] shows that $C_{i}$ are approximations of the balls $B_{i}$, and $D_{i}$ are also approximations in a weaker sense.

Lemma 8 (Chakrabarti and Regev [10]) The following events hold simultaneously with probability at least $3 / 4$ :

1. $B_{i} \subseteq C_{i} \subseteq B_{i+1}$ for all $i$.

2. For all $0 \leq j \leq i \leq\left\lceil\log _{\alpha} d\right\rceil$, at most a fraction $n^{-1 / s}$ of $B_{j}$ is not in $D_{i, j}$ and at most a fraction $n^{-1 / s}$ of $C_{i} \backslash B_{j+1}$ is in $D_{i, j}$.

\subsection{A simple $k$-round protocol for ANNS}

Theorem 9 (Theorem 2, restated) Let $\gamma>1$ be any constant. For $n>d$ and $k \geq 1$, ANNS $_{d, n}^{\gamma}$ has a $k$-round randomized cell-probing scheme with $O\left(k(\log d)^{1 / k}\right)$ cell-probes, table size $n^{O(1)}$ and word size $O(d)$.

As mentioned earlier, the solution will be presented as a public-coin cell-probing scheme, which by Proposition [ is then transformed to a standard randomized cell-probing scheme with the same cell-probe complexity and number of rounds on a table of polynomial size.

Without lost of generality, assume that $\gamma<4$ and let $\alpha \triangleq \sqrt{\gamma}$. Let $x \in\{0,1\}^{d}$ denote the query point and $B \subseteq\{0,1\}^{d},|B|=n$, denote the database. Recall that $B_{i}$, as defined in 11, are the sets of all database points within distance $\alpha^{i}$ of $x$.

There are two degenerate cases. The first case is when $B_{0}$ is not empty, which means $x \in B$. This case can be solved as a membership query of $x$ in the set $B$, by the perfect hashing with 1 cell-probe to a table of size $O\left(n^{2}\right)$, with the random hash function as public randomness. The second degenerate case is when $B_{1}$ is not empty, which means the query point $x$ is within distance 1 from $B$. This can also be solved as a membership query of $x$ in the 1-neighborhood $N_{1}(B)=$ $\left\{y \in\{0,1\}^{d} \mid \exists z \in B\right.$, dist $\left.(y, z) \leq 1\right\}$ of $B$, which contains at most $(d+1) n$ points, by the same method, using 1 cell-probe to a table of quadratic size with public randomness.

Note that these two instances of perfect hashing can run separately and in parallel to each other, and to the main data structure solving the non-degenerate cases, so that if a query $x$ finds itself within $B$ or within distance 1 from $B$, then the algorithm terminates and outputs the nearest neighbor. This will cost a polynomial addition to the table size and 2 more queries in the first round, but make no change to the number of rounds. For the rest, we can assume the following.

Assumption $1 B_{0}=B_{1}=\emptyset$. 
The goal of the main data structure is to find an $i$ such that $B_{i}$ is empty but $B_{i+2}$ is not and output a point in $B_{i+2}$, assuming that $B_{0}=B_{1}=\emptyset$. Such a point is clearly a $\gamma$-approximate nearest neighbor of $x$.

For $0 \leq i \leq\left\lceil\log _{\alpha} d\right\rceil$, let $M_{i}$ be the random $\left(c_{1} \log n\right) \times d$ matrices sampled independently as in Definition 7 and $C_{i} \subseteq B$ the subsets of database points constructed from $M_{i}$ as in Definition 7 , The random matrices $M_{i}$ are treated as the public randomness shared between the cell-probing algorithm and the table. The table who possesses the database $B$ may construct $C_{i}$ from $M_{i} x$ for every possible $x \in\{0,1\}^{d}$ (in fact, for every $M_{i} x \in\{0,1\}^{c_{1} \log n}=\left[n^{c_{1}}\right]$ ), while the cell-probing algorithm who possesses the query point $x$ may compute the product $M_{i} x$ from the actual query point $x$.

By Lemma 8, the following assumption holds with probability at least 3/4:

Assumption $2 B_{i} \subseteq C_{i} \subseteq B_{i+1}$ for all $i$.

With this assumption, the algorithm only needs to find an $i$ such that $C_{i} \neq \emptyset$ but $C_{i-1}=\emptyset$. Since $B_{i-1} \subseteq C_{i-1}=\emptyset$ and $B_{i+1} \supseteq C_{i} \neq \emptyset$, any point in $C_{i}$ is a $\gamma$-approximation nearest neighbor of $x$.

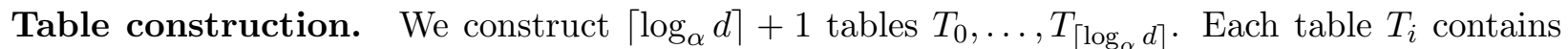
$2^{c_{1} \log n}=\operatorname{poly}(n)$ many cells, where each cell corresponds to a string $j \in\{0,1\}^{c_{1} \log n}$, so the total number of cells in all these tables is a polynomial of $n$. Here $c_{1}$ is the constant factor in the number of rows of $M_{i}$. Due to the public randomness, the table contents may depend on both the database $B$ and the public random matrices $M_{i}$.

For every $0 \leq i \leq\left\lceil\log _{\alpha} d\right\rceil$ and every $j \in\{0,1\}^{c_{1} \log n}$, the content of the $j$-th cell $T_{i}[j]$ in the $i$-th table $T_{i}$ is given as follows:

- If there exists a datapoint $z \in B$ such that $\operatorname{dist}\left(j, M_{i} z\right) \leq \delta\left(\alpha^{i}, \alpha\right) \cdot c_{1} \log n$, the cell $T_{i}[j]$ stores an arbitrary one of such $z$.

- If otherwise there is no such datapoint, $T_{i}[j]$ stores a special symbol indicating the EMPTY.

Note that $M_{i} x \in\{0,1\}^{c_{1} \log n}$ is a valid address for the cells in a table $T_{i}$. And for every $i$, the table cell $T_{i}\left[M_{i} x\right]$ stores a point from $C_{i}$ if $C_{i}$ is not empty, or $T_{i}\left[M_{i} x\right]=$ EMPTY if $C_{i}=\emptyset$.

Cell-probing algorithm. The algorithm possesses the query point $x$ and the public random matrices $M_{i}$. Set $\tau=c^{\prime}(\log d)^{1 / k}$, for a constant $c^{\prime} \geq \log _{\alpha} 4$ so that

$$
\tau \cdot\left(\frac{\tau}{2}\right)^{k-1} \geq\left\lceil\log _{\alpha} d\right\rceil
$$

The cell-probing algorithm consists of at most $(k-1)$ shrinking rounds, succeeded by one final completion round. And if $k=1$, the algorithm is non-adaptive and just consists of a completion round. In every round the algorithm makes at most $\tau$ parallel cell-probes to the table. The total number of cell-probes is at most $(\tau-1)(k-1)+\tau=O\left(k\left(\log _{\alpha} d\right)^{1 / k}\right)$. The pseudocode of the cell-probing algorithm is given in Algorithm 1.

The algorithm finds a $\gamma$-approximate nearest neighbor of $x$ by a multi-way search: it maintains two integers $l$ and $u$, initially $l=0$ and $u=\left\lceil\log _{\alpha} d\right\rceil$. At each round $l$ and $u$ are updated, satisfying the invariant that $l<u, C_{l}=\emptyset$ and $C_{u} \neq \emptyset$. This invariant is satisfied initially since by

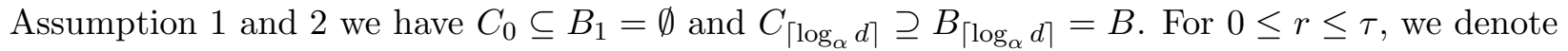
$\rho(r) \triangleq\left\lfloor l+\frac{r}{\tau}(u-l)\right\rfloor$. The cell-probing algorithm proceeds as follows: 


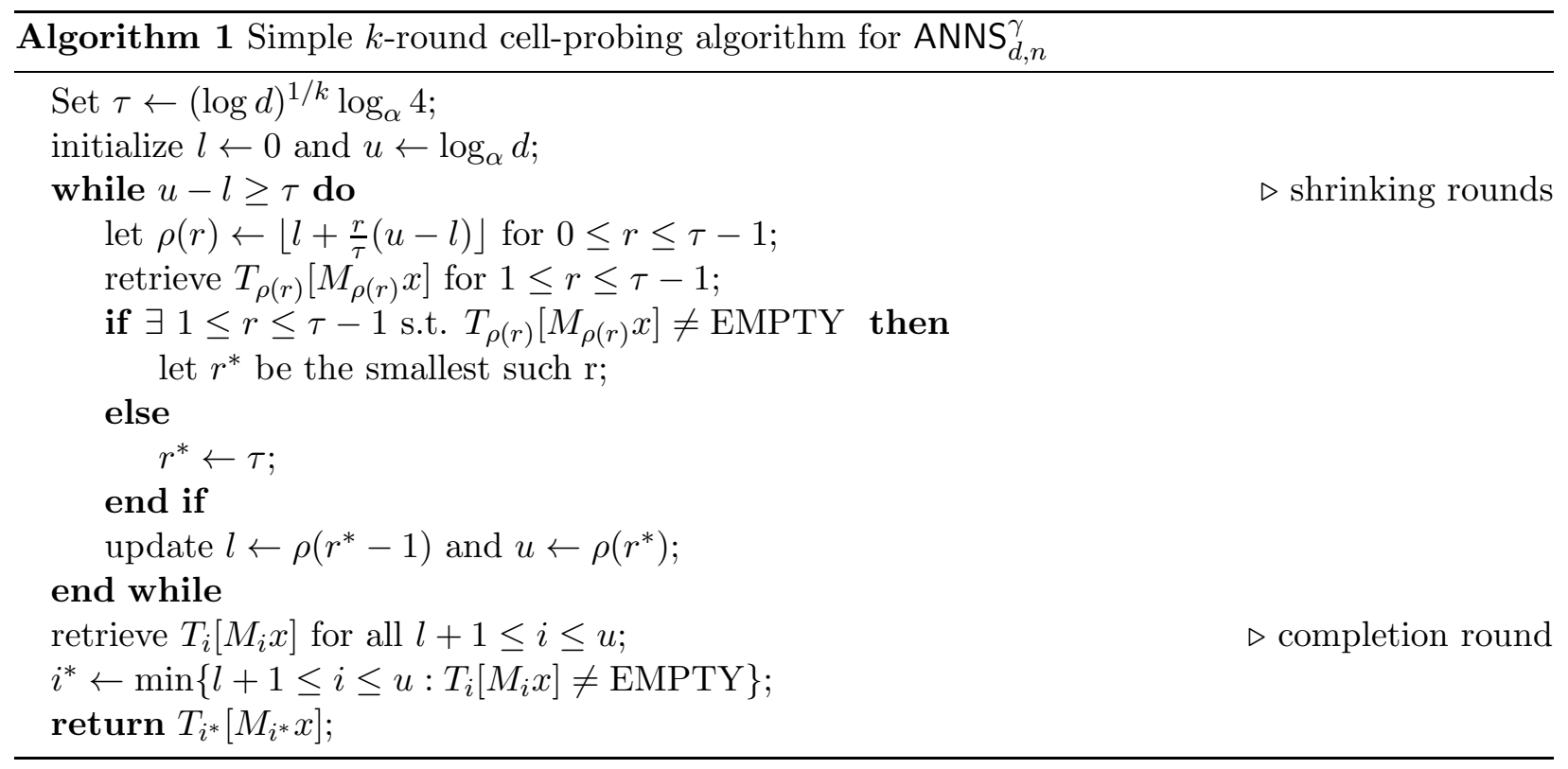

1. In each shrinking round: the algorithm reads the contents of $T_{\rho(r)}\left[M_{\rho(r)} x\right]$ for all $1 \leq r \leq \tau-1$ in parallel, and finds those $r$ such that $T_{\rho(r)}\left[M_{\rho(r)} x\right] \neq$ EMPTY, which means $C_{\rho(r)} \neq \emptyset$. Let $r^{*}$ be the smallest such $r$, or let $r^{*}=\tau$ if no such $r$ exists. Update $l$ to $\rho\left(r^{*}-1\right)$ and $u$ to $\rho\left(r^{*}\right)$. The new gap between $l$ and $u$ is $\rho\left(r^{*}\right)-\rho\left(r^{*}-1\right)$, which is at most $(u-l) / \tau+1$.

2. Once the gap $u-l$ drops below $\tau$, the algorithm enters the completion round: it reads the cells $T_{i}\left[M_{i} x\right]$ for all $l+1 \leq i \leq u$ in parallel, finds the smallest $i$ such that $T_{i}\left[M_{i} x\right] \neq$ EMPTY, and outputs the point stored in that cell. Such $i$ must exist since we know $C_{u} \neq \emptyset$. Note that the output point is from a nonempty $C_{i}$ such that $C_{i-1}=\emptyset$. With Assumption 2, it must be a $\gamma$-approximate nearest neighbor of $x$.

Note that in every shrinking round, $l$ and $u$ are updated to $l^{\prime}$ and $u^{\prime}$ respectively so that $u^{\prime}-l^{\prime} \leq$ $(u-l) / \tau+1 \leq 2(u-l) / \tau$ as long as $u-l \geq \tau$. And once $u-l<\tau$, the algorithm enters the completion round. Recall that $\tau \cdot(\tau / 2)^{k-1} \geq\left\lceil\log _{\alpha} d\right\rceil$. Hence, there can be at most $(k-1)$ shrinking rounds.

\subsection{A $k$-round protocol for ANNS for large $k$}

Theorem 10 (Theorem [3, restated) Let $\gamma>1$ and $c>2$ be any constants. For $n>d$ and $k>5 c^{2} /(c-2)$, ANNS $_{d, n}^{\gamma}$ has a k-round randomized cell-probing scheme with $O\left(k+\left(\frac{1}{k} \log d\right)^{c / k}\right)$ cell-probes, table size $n^{O(1)}$, and word size $O(d)$.

As before the algorithm is also presented as a public-coin cell-probing scheme, and is transformed into a standard randomized cell-probing scheme by Proposition 6.

This more sophisticated algorithm reuses several components of the simple algorithm in Theorem 9. For $0 \leq i \leq\left\lceil\log _{\alpha} d\right\rceil$, the sets $B_{i}$ and $C_{i}$, and the random matrices $M_{i}$ are constructed in the same way as before. The degenerate cases when $B_{0}$ or $B_{1}$ is not empty are also handled in the same as before, so we proceed by assuming Assumption 1 . 
Set $s \triangleq\left(\frac{1}{4}-\frac{1}{2 c}\right) k-\frac{1}{4}>1$. We assume that $k=o(\ln \ln d)$, because for some sufficiently large $k=O(\ln \ln d / \ln \ln \ln d)$, it can be verified that our algorithm already makes $O(1)$ cell-probes per round on average, so there is no need to consider larger number of rounds after that. Hence, we have $1<s<\ln \ln d<\ln \ln n$.

Let $N_{j}$ be the random $\left(\frac{c_{2}}{s} \log n\right) \times d$ matrices sampled independently as in Definition 7 and $D_{i, j} \subseteq B$ the subsets of database points constructed from $M_{i}$ and $N_{j}$ as in Definition 7 . Now the public randomness shared between the cell-probing algorithm and the table are the random matrices $M_{i}$ and $N_{j}$ for $0 \leq j \leq i \leq\left\lceil\log _{\alpha} d\right\rceil$. We make another assumption.

Assumption 3 For all $0 \leq j \leq i \leq\left\lceil\log _{\alpha} d\right\rceil$, at most a fraction $n^{-1 / s}$ of $B_{j}$ is not in $D_{i, j}$ and that at most a fraction $n^{-1 / s}$ of $C_{i} \backslash B_{j+1}$ is in $D_{i, j}$.

By Lemma 8, the error probability of an algorithm that succeeds by assuming both Assumption 2 and Assumption 3 is at most $1 / 4$.

Table construction. We reuse the $\left\lceil\log _{\alpha} d\right\rceil+1$ tables $T_{0}, \ldots, T_{\left\lceil\log _{\alpha} d\right\rceil}$ constructed in Theorem 9 , In addition, we further construct $\left(\left\lceil\log _{\alpha} d\right\rceil+1\right) \times 2^{c_{1} \log n}$ auxiliary tables $\widetilde{T}_{i, j}$ for $0 \leq i \leq\left\lceil\log _{\alpha} d\right\rceil$ and $j \in\{0,1\}^{c_{1} \log n}$. The address of each cell in an auxiliary table $\widetilde{T}_{i, j}$ corresponds to a concatenation $\bar{w}=\left\langle l, u, w_{0}, w_{1}, \ldots, w_{s}\right\rangle$ of:

- a pair of lower and upper thresholds $0 \leq l \leq u \leq\left\lceil\log _{\alpha} d\right\rceil$;

- a special index $1 \leq w_{0} \leq s$

- $s$ short strings $w_{1}, \ldots, w_{s} \in\{0,1\}^{\frac{c_{2}}{s} \log n}$.

Altogether these correspond to at most $\left(\log _{\alpha} d\right) s 2^{c_{2} \log n}=\operatorname{poly}(n)$ cells in each auxiliary table. The total number of cells in all tables remains to be a polynomial of $n$.

For $0 \leq i \leq\left\lceil\log _{\alpha} d\right\rceil, j \in\{0,1\}^{c_{1} \log n}$, and any address $\bar{w}=\left\langle l, u, w_{0}, w_{1}, \ldots, w_{s}\right\rangle$ of cells in auxiliary table $\widetilde{T}_{i, j}$, the content of the cell $\widetilde{T}_{i, j}[\bar{w}]$ is given as follows: For $1 \leq r \leq s$, define $\rho(r) \triangleq\left\lfloor l+\frac{r-1}{s-1}(u-l)\right\rfloor$. Let $j=M_{i} x$ and $w_{r}=N_{\rho(r)} x$ for $1 \leq r \leq s$. We construct the sets $C_{i}, D_{i, \rho(1)}, \ldots, D_{i, \rho(s)}$ since we now have complete information about the sets.

- If there exists an $1 \leq r \leq w_{0}$ such that $\left|D_{i, \rho(r)}\right|>n^{-1 / s}\left|C_{i}\right|$, then the cell $\widetilde{T}_{i, j}[\bar{w}]$ stores the smallest such $r$.

- If otherwise there is no such $r$, the cell $\widetilde{T}_{i, j}[\bar{w}]$ stores $s+1$.

Cell-probing algorithm. Set $\tau=c^{\prime}\left(\frac{1}{k} \log d\right)^{c / k}$ for some constant $c^{\prime} \geq \log _{\alpha} 4$ so that

$$
\left(\frac{\tau}{2}\right)^{\frac{k-1}{2}-2 s} \geq\left\lceil\frac{\log _{\alpha} d}{k}\right\rceil
$$

The cell-probing algorithm contains at most $(k-1) / 2$ shrinking phases, succeeded by one final completion round. Each shrinking phase contains at most two rounds. In every shrinking phase the 
algorithm makes at most $\frac{\tau-1}{s}+2$ cell-probes to the table, and in the completion round it makes at most $\max \{3 \tau, k\}$ parallel cell-probes. Thus the total number of cell-probes is at most

$$
\frac{k-1}{2}\left(\left\lceil\frac{\tau-1}{s}\right\rceil+2\right)+\max (3 \tau, k)=O\left(k+\left(\frac{1}{k} \log _{\alpha} d\right)^{c / k}\right) .
$$

The algorithm maintains two integers $l$ and $u$, initially $l=0$ and $u=\left\lceil\log _{\alpha} d\right\rceil$. At each shrinking phase $l$ and $u$ are updated, satisfying the invariant that $l<u, C_{l}=\emptyset$ and $C_{u} \neq \emptyset$. This invariant is satisfied initially since we have $C_{0} \subseteq B_{1}=\emptyset$ and $C_{\left\lceil\log _{\alpha} d\right\rceil} \supseteq B_{\left\lceil\log _{\alpha} d\right\rceil}=B$.

The aim of the algorithm is at each shrinking phase to shrink the gap $u-l$ by a factor of $O(\tau)$ or to shrink the size of $C_{u}$. When the gap $u-l$ drops below $\max \{3 \tau, k\}$ the algorithm enters the completion round, where sets $C_{l}, \ldots, C_{u}$ are searched simultaneously by at most $\max \{3 \tau, k\}$ parallel cell-probes in one round. We claim that at each shrinking phase, the algorithm updates $l$ and/or $u$ in such a way that either $u^{\prime}-l^{\prime} \leq(u-l) / \tau+3$ or $\left|C_{u^{\prime}}\right| \leq n^{-1 / 2 s}\left|C_{u}\right|$, where $l^{\prime}$ and $u^{\prime}$ denote the updated values of $l$ and $u$, respectively.

For $0 \leq r \leq \tau-1$, we denote $\rho(r) \triangleq\left\lfloor l+\frac{r}{\tau}(u-l)\right\rfloor$. The cell-probing algorithm proceeds as follows:

1. In shrinking phase: among sets $D_{u, \rho(1)}, \ldots, D_{u, \rho(\tau-1)}$, the algorithm will first find the smallest $r$ such that $\left|D_{u, \rho(r)}\right|>n^{-1 / s}\left|C_{u}\right|$. To find such $r$, the algorithm first arranges these sets into $\lceil(\tau-1) / s\rceil$ groups where each group contains up to at most $s$ sets, with each group consumes one parallel cell-probe as follows: for every $1 \leq j \leq\lceil(\tau-1) / s\rceil$, let the concatenation $\bar{w}^{j}=\left\langle l_{j}, u_{j}, w_{0}^{j}, w_{1}^{j}, \ldots, w_{s}^{j}\right\rangle$ be constructed as:

- the lower and upper thresholds for the current group: $l_{j}=\rho(1+(j-1) s)$ and $u_{j}=\rho(j s)$;

- $w_{0}^{j}$ gives the number of sets $D_{u, \rho(r)}$ in the current group: normally it is just $s$ except for the last group due to the rounding, so $w_{0}^{j}=\tau-1-s\left\lfloor\frac{\tau-1}{s}\right\rfloor$ if $j=\lceil(\tau-1) / s\rceil$ and $s \nmid \tau-1$, and $w_{0}^{j}=s$ if otherwise;

- for $q=1,2, \ldots, w_{0}^{j}$, let $w_{q}^{j}=N_{\rho(1+(j-1) s+q-1)} x$.

The algorithm reads the contents of cells $T_{u}\left[M_{u} x\right]$ and $\widetilde{T}_{u, M_{u} x}\left[\bar{w}^{j}\right]$ for all $1 \leq j \leq\lceil(\tau-1) / s\rceil$ in parallel. Let $j^{*}$ be the smallest $j$ such that $\widetilde{T}_{u, M_{u} x}\left[\bar{w}^{j}\right] \neq s+1$, or $j^{*}=(\tau-1) / s+1$ if no such $j$ exists. If $j^{*}=(\tau-1) / s+1$, let $r^{*}=\tau$. Otherwise let $r^{*}=\left(j^{*}-1\right) s+\widetilde{T}_{u, M_{u} x}\left[\bar{w}^{j^{*}}\right]$. Remember that $\widetilde{T}_{u, M_{u} x}\left[\bar{w}^{j}\right]=s+1$ means that all $\left|D_{u, l_{j}}\right|, \ldots,\left|D_{u, \rho\left(1+(j-1) s+w_{0}-1\right)}\right| \leq n^{-1 / s}\left|C_{u}\right|$. Hence such $r^{*}$ is the smallest $r \in[\tau]$ such that $\left|D_{u, \rho(r)}\right|>n^{-1 / s}\left|C_{u}\right|$, or $r^{*}=\tau$ if no such $r$ exists. There are three cases:

(a) If $r^{*}=1$ (CASE 1 ), the algorithm updates $u$ to $\rho(1)+1$, leaving $l$ unchanged, skips the second round and moves to the next phase.

(b) Otherwise, the algorithm reads the content of cell $T_{\rho\left(r^{*}-1\right)-1}\left[M_{\rho\left(r^{*}-1\right)-1} x\right]$. If the cell is EMPTY (CASE 2), it updates $l$ to $\rho\left(r^{*}-1\right)-1$ and if further $r^{*}<\tau$, updates $u$ to $\rho\left(r^{*}\right)+1$.

(c) If $T_{\rho\left(r^{*}-1\right)-1}\left[M_{\rho\left(r^{*}-1\right)-1} x\right] \neq \operatorname{EMPTY}(\mathrm{CASE} 3)$, the algorithm updates $u$ to $\rho\left(r^{*}-1\right)-1$, leaving $l$ unchanged. 


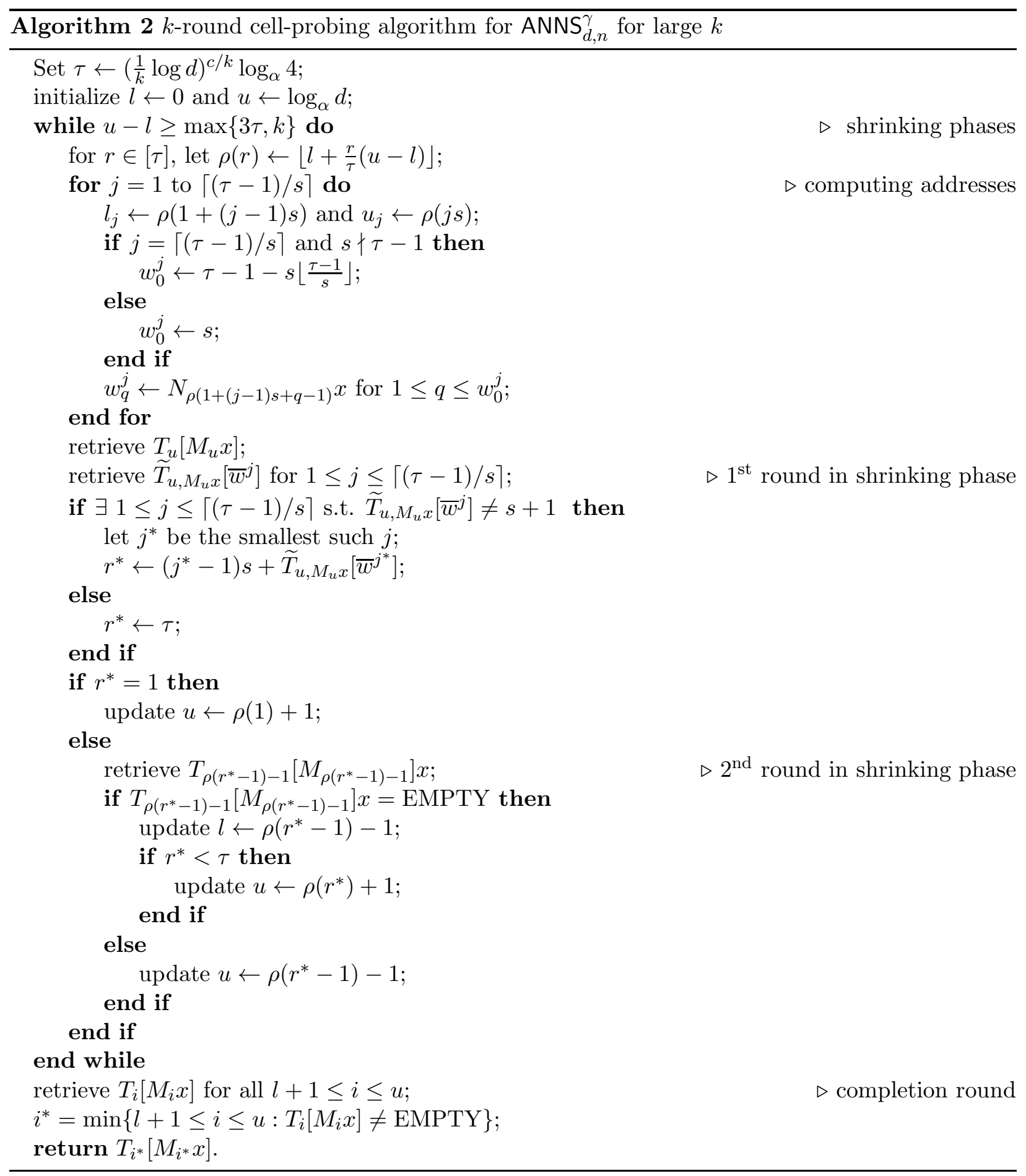


2. Once the gap $u-l$ drops below $\max \{3 \tau, k\}$, the algorithm enters the completion round: it reads the cells $T_{i}\left[M_{i} x\right]$ for all $l+1 \leq i \leq u$ in parallel, finds the smallest $i$ such that $T_{i}\left[M_{i} x\right] \neq$ EMPTY, and outputs the point stored in that cell. Such $i$ must exist since we know $C_{u} \neq \emptyset$. Note that the output point is from a nonempty $C_{i}$ such that $C_{i-1}=\emptyset$. With Assumption 2, it must be a $\gamma$-approximate nearest neighbor of $x$.

The pseudocode of the cell-probing algorithm is given in Algorithm 2 ,

We now verify that at each time when the $l$ and $u$ are updated, the invariant that $l<u, C_{l}=\emptyset$ and $C_{u} \neq \emptyset$ is satisfied. First, in all three cases $l<u$ is obviously satisfied after update.

- Since in CASE 1 and CASE 3 the lower threshold $l$ is not changed, $C_{l}$ stays empty. And in CASE 2, $T_{\rho\left(r^{*}-1\right)-1}\left[M_{\rho\left(r^{*}-1\right)-1} x\right]=$ EMPTY implies that the set $C_{l}=C_{\rho\left(r^{*}-1\right)-1}$ is empty.

- In CASE 3 , since $T_{\rho\left(r^{*}-1\right)-1}\left[M_{\rho\left(r^{*}-1\right)-1} x\right] \neq$ EMPTY, the set $C_{u}=C_{\rho\left(r^{*}-1\right)-1}$ is nonempty. In CASE 2 when $r^{*}=\tau$ the upper threshold $u$ is not changed so that $C_{u}$ stays nonempty. For the remaining cases, since $\left|D_{u, \rho\left(r^{*}\right)}\right|>n^{-1 / s}\left|C_{s}\right|$, by Assumption 3, the set $D_{u, \rho\left(r^{*}\right)}$ must contains at least one point from $B_{\rho\left(r^{*}\right)+1}$. Since $B_{\rho\left(r^{*}\right)+1} \subseteq C_{\rho\left(r^{*}\right)+1}$, the set $C_{u}=C_{\rho\left(r^{*}\right)+1}$ is nonempty.

And note that in CASE 1 and CASE 2, the gap between the updated values of $l$ and $u$ is at most $\left(\left\lfloor l+\frac{r^{*}}{\tau}(l-u)\right\rfloor+1\right)-\left(\left\lfloor l+\frac{r^{*}-1}{\tau}(l-u)\right\rfloor-1\right) \leq \frac{l-u}{\tau}+3$, and in CASE 3, the size of the new $C_{u}$ is $\left|C_{\rho_{r^{*}-1}-1}\right| \leq\left|B_{\rho_{r^{*}-1}}\right| \leq\left|D_{u, \rho_{r^{*}-1}}\right| /\left(1-n^{-1 / s}\right) \leq 2\left|D_{u, \rho_{r^{*}-1}}\right| \leq 2 n^{-1 / s}\left|C_{u}\right|$. Therefore, in each shrinking phase, either $u^{\prime}-l^{\prime} \leq(u-l) / \tau+3$ or $\left|C_{u^{\prime}}\right| \leq n^{-1 / 2 s}\left|C_{u}\right|$, where $l^{\prime}$ and $u^{\prime}$ denote the updated values of $l$ and $u$, respectively.

Notice that as $C_{u}$ stays nonempty, there are most $2 s$ shrinking phases in which $\left|C_{u}\right|$ drops. On the other hand, as long as $u-l \geq \max (3 \tau, k)$, we have $(u-l) / \tau+3 \leq 2(u-l) / \tau$. Since we choose our $\tau$ to satisfy $\frac{\log _{\alpha} d}{k} \leq(\tau / 2)^{(k-1) / 2-2 s}$, there can be at most $(k-1) / 2-2 s$ shrinking phases in which $(u-l)$ shrinks by a factor of $2 / \tau$. Hence, overall there can be at most $(k-1) / 2$ shrinking phases. Each shrinking phase contains at most 2 rounds, where the algorithm makes $\left\lceil\frac{\tau-1}{s}\right\rceil+1$ parallel cell-probes in the first round and one cell-probe in the second round of that phase, and at last in the completion round the algorithm makes $\max (3 \tau, k)$ parallel cell-probes. The total number of cell-probes is as given by (4).

\subsection{A 1-probe protocol for $\lambda$-ANN}

The algorithms presented in previous sections are for the search of the nearest neighbors. These highlighted words seem to be critical to this non-trivial cell-probe complexity on a table of polynomial size when both randomization and approximation are allowed.

Consider a well-known decision version of the problem: the approximate $\lambda$-near neighbor problem $\lambda$-ANN ${ }_{d, n}^{\gamma}$. Let $\gamma>1, \lambda>0$ be fixed. A point $y \in\{0,1\}^{d}$ is a $\lambda$-near neighbor to $x \in\{0,1\}^{d}$ if $\operatorname{dist}(x, y) \leq \lambda$. Given a query point $x \in\{0,1\}^{d}$ and a database $B \subseteq\{0,1\}^{d}$, the problem $\lambda$-ANN ${ }_{d, n}^{\gamma}$ asks to distinguish between the two cases: (1) there is a database point $y \in B$ which is a $\lambda$-near neighbor to $x$, and (2) there is no database point $y \in B$ which is a $\gamma \lambda$-near neighbor to $x$. For other cases, the answer can be arbitrary. This problem has been extensively studied in the context of lower bounds for nearest neighbor search [6, ,7, 16, 20, 21].

The following is a folklore result: if randomization is allowed then $\lambda$ - $\mathrm{ANN}_{d, n}^{\gamma}$ can be solved with 1-probe on a table of polynomial size. We actually show this for a slightly stronger search problem, 
the approximate $\lambda$-near neighbor search problem $\lambda$-ANNS ${ }_{d, n}^{\gamma}$, where if it is the case that there is a $\lambda$-near neighbor in the database, a database point which is a $\gamma \lambda$-near neighbor is output.

Theorem 11 Let $\gamma>1$ be any constant. For $n>d, \lambda$-ANNS ${ }_{d, n}^{\gamma}$ has a randomized cell-probing scheme for with 1 cell-probe, table size $n^{O(1)}$, and word size $O(d)$.

Proof: Here we still present a public-coin cell-probing scheme. Apparently the same generic translation in Proposition 6 also holds for the $\lambda$-ANNS ${ }_{d, n}^{\gamma}$ problem.

Still let $\alpha=\sqrt{\gamma}$. The table is prepared precisely as in Theorem 9, with the public random matrices $M_{i}$ shared between the cell-probing algorithm and the table, and the points from sets $C_{i}$ which approximate the balls $B_{i}$ of database points storing in the table.

For the cell-probing algorithm, let $i=\left\lceil\log _{\alpha} \lambda\right\rceil$. Thus $\alpha^{i} \geq \lambda$ and $\alpha^{i+1} \leq \gamma \lambda$. The cell-probing algorithm reads the cell $T_{i}\left[M_{i} x\right]$ returns the content if it contains a point or returns a NO if it is EMPTY. As argued before, this cell stores a point from $C_{i}$ if $C_{i}$ is not empty. Note that if there exist database points which are within distance $\lambda$ from $x$, then $B_{i}$ is not empty. By Assumption 2 , $B_{i} \subseteq C_{i} \subseteq B_{i+1}$, thus $C_{i}$ is not empty. In this case a point in $C_{i}$ must be returned, which is a $\gamma \lambda$-near neighbor to $x$. If no database point is a $\gamma \lambda$-near neighbor to $x$, then $B_{i+1}$ is empty, and due to Assumption 2, so is $C_{i}$, therefore the algorithm may only find $T_{i}\left[M_{i} x\right]=$ EMPTY and return with a NO.

\section{Lower Bounds}

In this section, we prove the following lower bound for $k$-round randomized approximate nearest neighbor search.

Theorem 12 (Theorem 4, restated) For any finite $c_{1}, c_{2}>0$, there exists a $c_{3}>0$ such that the following holds. Let $n, d \geq 1$ be sufficiently large integers such that $d \leq 2^{\sqrt{\log n}}$ and $n \leq 2^{d^{0.99}}$. Let $1 \leq k \leq \frac{\log \log d}{2 \log \log \log d}$ be an integer. If $\mathrm{ANNS}_{d, n}^{\gamma}$ has a $k$-round randomized cell-probing scheme with table size $s \leq n^{c_{1}}$, word size $w \leq d^{c_{2}}$, such that every query is correctly answered within $t$ total cell-probes in $k$ rounds with probability at least $7 / 8$, then $t>\frac{c_{3}}{k}\left(\log _{\gamma} d\right)^{1 / k}$.

The proof follows the framework given in [10]. The framework consists of three main components:

1. A reduction from $\operatorname{LPM}_{m, n}^{\Sigma}$ to $\mathrm{ANNS}_{d, n}^{\gamma}$ : As observed by Theorem 11, it is impossible to prove the lower bound by considering the decision version of $\operatorname{ANNS}_{d, n}^{\gamma}$. The longest prefix match problem $\mathrm{LPM}_{m, n}^{\Sigma}$ captures the nature of $\mathrm{ANNS}_{d, n}^{\gamma}$ very well, and meanwhile, is convenient for applying the round eliminations.

2. A round elimination lemma for communication protocols for $\operatorname{LPM}_{m, n}^{\Sigma}$ : Cell-probing schemes are represented as communication protocols. Eliminating a round in any communication protocol for $\mathrm{LPM}_{m, n}^{\Sigma}$ gives a weaker protocol for the same problem of a smaller scale.

3. Applying the round elimination to $\mathrm{LPM}_{m, n}^{\Sigma}$ until there is no round left yet the problem is still nontrivial. 
Here a simple observation for the $k$-round cell-probing schemes is that $k$ rounds of cell-probes can be simulated by $2 k$ rounds of communications. Applying the above framework with this observation, for the first two component, we redo the reduction with a new choice of parameters, and reprove the round elimination lemma for general communication protocols with non-uniform message sizes in different rounds.

In fact, these variations can be handled routinely by carefully going through the original proofs with new parameters and/or more generic settings. The most delicate part of our lower bound is our execution of the third step in above framework, which involves an exploitation of the power of round eliminations. This part is in the proof of our main lower bound Theorem 24.

\subsection{Reduction from longest prefix match}

In [10], a reduction from another data structure problem, the longest prefix matching $\operatorname{LPM}_{m, n}^{\Sigma}$, to ANNS $_{d, n}^{\gamma}$ is constructed.

Definition 13 (longest prefix match) For integers $m, n \geq 1$ and a finite alphabet $\Sigma$ we define the longest prefix match problem $\mathrm{LPM}_{m, n}^{\Sigma}$ as the data structure problem that given a query $x \in \Sigma^{m}$ and a database $B \subseteq \Sigma^{m},|B|=n$, an answer $z \in B$ must be returned to satisfy that $z$ has the longest common prefix with $x$ among all $y \in B$.

The reduction in [10] maps instances of $\mathrm{LPM}_{m, n}^{\Sigma}$ to instances of $\mathrm{ANNS}_{d, n}^{\gamma}$ without going through the computation model, so it also applies to $k$-round cell-probing schemes. In order to prove our more refined lower bound, we need to guarantee the same reduction to hold for a more critical parameterization.

Fix the parameters for the problem $\operatorname{ANNS}_{d, n}^{\gamma}$. We define $\eta$ and $\beta$ as follows:

$$
\eta \triangleq 1-\frac{\log \log \gamma}{\log \log d} \quad \text { and } \quad \beta \triangleq 1-\frac{c_{4}}{\log \log d},
$$

where $c_{4}=2 \log 201$. Note that it holds that

$$
\gamma=2^{(\log d)^{1-\eta}} .
$$

Lemma 14 (reduction from $\mathrm{LPM}_{m, n}^{\Sigma}$ to $\mathrm{ANNS}_{d, n}^{\gamma}$ ) Let $d$ be a sufficiently large integer, let $\eta$ and $\beta$ be as defined in (5) so that $\gamma$ satisfies (6) , and set $m \triangleq\left\lfloor(\log d)^{\eta \beta}\right\rfloor$. Let $\Sigma$ be an alphabet of size $\left\lceil 2^{d^{0.99}}\right\rceil$. If $\mathrm{ANNS}_{d, n}^{\gamma}$ has a k-round randomized cell-probing scheme with cell-probe complexity $t$ and success probability $7 / 8$, using table size $s$ and word size $w$, then so does $\operatorname{LPM}_{m, n}^{\Sigma}$.

Next we explain how to modify the reduction in [10 to prove this lemma.

A family of Hamming balls in $\{0,1\}^{d}$ is said to be $\gamma$-separated if the distance between any two points belonging to distinct balls in the family is more than $\gamma$ times the diameter of any ball in the family. The following lemma is due to Chakrabarti et al. [9].

Lemma 15 (rephrased from Lemma 3.2 in [9]) Let $d \geq 1$ be a large enough integer, and let $\gamma>1$. Inside a Hamming ball of radius $r$ (where $\left.d^{0.995} \leq r \leq d\right)$ in $\{0,1\}^{d}$ there exists a $\gamma$-separated family of $\left\lceil 2^{d^{0.99}}\right\rceil$ balls, each of radius $r /(8 \gamma)$. 
Lemma 16 (improved from Lemma 2.3 in [10]) Let $d \geq 1$ be a large enough integer, and let $\gamma=2^{(\log d)^{1-\eta}} \geq 3$, as defined in (6). There exists a rooted tree $\mathcal{T}$ whose vertices are Hamming balls in $\{0,1\}^{d}$ and which satisfies the following properties:

1. If $v$ is a child of $u$ in $\mathcal{T}$, then as Hamming balls $v \subset u$.

2. Each non-leaf vertex of $\mathcal{T}$ has exactly $\left\lceil 2^{d^{0.99}}\right\rceil$ children.

3. Each depth-i vertex (the root being a depth-0 vertex) has radius $d /(8 \gamma)^{i}$.

4. The depth-i vertices form a $\gamma$-separated family of Hamming balls, which means the distance between any two points belonging to distinct balls in the family is more than $\gamma$ times the diameter of any ball in the family.

5. The leaves of $\mathcal{T}$ are at depth $\left\lfloor(\log d)^{\eta \beta}\right\rfloor$, where $\eta$ is as defined in (5) $)$.

Proof: The proof is almost identical to the proof of Lemma 2.3 in [10, which follows a construction due to Chakrabarti et al. 9]. Note that the balls at leaves have radius of at least $d /(8 \gamma)^{\left\lfloor(\log d)^{\eta \beta}\right\rfloor}$. By our choices of $\eta$ and $\beta$ as defined in (5), it can be verify that for large enough $d$,

$$
\frac{d}{(8 \gamma)^{\left\lfloor(\log d)^{\eta \beta}\right\rfloor}} \geq d^{0.995}
$$

Then by Lemma 15, we have the suitable tree $\mathcal{T}$ by a natural recursive construction.

Given this tree $\mathcal{T}$, the reduction from $\operatorname{LPM}_{m, n}^{\Sigma}$ with the new string length $m=\left\lfloor(\log d)^{\beta \eta}\right\rfloor$ to ANNS $_{d, n}^{\gamma}$ can be constructed by reusing the mapping from $\mathrm{LPM}_{m, n}^{\Sigma}$ instances to $\mathrm{ANNS}_{d, n}^{\gamma}$ instances described in the proof of Lemma 2.4 in [10] as a blackbox.

\subsection{Round elimination for communication protocols}

We now consider communication protocols between two players Alice and Bob in Yao's model of communication complexity [23. We refer the readers to the nice textbook by Kushilevitz and Nisan [14] for formal definitions of various concepts, e.g. private-coin protocols.

We assume Alice and Bob send messages to each other alternatively. We use two vectors $\mathbf{A}=\left(a_{1}, a_{2}, \ldots, a_{k}\right)$ and $\mathbf{B}=\left(b_{1}, b_{2}, \ldots, b_{k}\right)$ to respectively denote the lengths of messages sent by Alice and Bob in each round.

Definition 17 Let $\mathbf{A}=\left(a_{1}, a_{2}, \ldots, a_{k}\right) \in \mathbb{R}_{>0}^{k}$ and $\mathbf{B}=\left(b_{1}, b_{2}, \ldots, b_{k}\right) \in \mathbb{R}_{\geq 0}^{k}$. An $\langle\mathbf{A}, \mathbf{B}, 2 k\rangle^{A_{-}}$ protocol is a $2 k$-round communication protocol, in which Alice and Bob send messages to each other alternatively, with Alice sending the first message, with the size of Alice's $i$-th message being exactly $\left\lfloor a_{i}\right\rfloor$ bits, and the size of Bob's $i$-th message being exactly $\left\lfloor b_{i}\right\rfloor$ bits. The superscript " $A$ " indicates that Alice sends the first message.

For $\mathbf{A}=\left(a_{1}, \ldots, a_{k-1}\right) \in \mathbb{R}_{\geq 0}^{k-1}$ and $\mathbf{B}=\left(b_{1}, \ldots, b_{k}\right) \in \mathbb{R}_{\geq 0}^{k}$, we call such a protocol an $\langle\mathbf{A}, \mathbf{B}, 2 k-1\rangle^{B}$-protocol if the first message is sent by Bob.

A data structure problem $\rho \subseteq \mathscr{A} \times \mathscr{B} \times \mathscr{C}$ is naturally a communication problem: Alice is give a query $x \in \mathscr{A}$ as input, Bob is given a database $B \in \mathscr{B}$ as input, and Alice is asked to output a correct answer $z \in \mathscr{C}$ satisfying $(x, B, z) \in \rho$ after communicating with Bob. As observed in [17], any cell-probing scheme is actually a communication protocol, with Alice being the cell-probing algorithm and Bob being the table. 
Proposition 18 If a data structure problem $\rho$ has a randomized cell-probing scheme using table size $s$ and word size $w$ bits, such that every query is answered correctly within t total cell-probes in $k$ rounds with probability $1-\epsilon$, then $\rho$ has a private-coin $\langle\mathbf{A}, \mathbf{B}, 2 k\rangle^{A}$-protocol with $a_{i}=t_{i}\lceil\log s\rceil$ and $b_{i}=t_{i} w$ for every $1 \leq i \leq k$, for some $t_{1}, t_{2}, \ldots, t_{k} \geq 0$ that $t=\sum_{i=1}^{k} t_{i}$, such that Alice outputs a correct answer with probability at least $1-\epsilon$.

Here the natural interpretation is that each round of $t_{i}$ many parallel cell-probes can be simulated by two rounds of communications: Alice sends the addresses of the $t_{i}$ cells, each of $\lceil\log s\rceil$ bits, to Bob, and Bob responds by sending back the contents of these $t_{i}$ cells, each of $w$ bits.

Let $\mathbf{A}=\left(a_{1}, a_{2}, \ldots, a_{k}\right)$ and $\mathbf{B}=\left(b_{1}, b_{2}, \ldots, b_{k^{\prime}}\right)$ be two vectors, and $c \in \mathbb{R}$ be a number. We introduce some notations:

- let $c \mathbf{A}=\left(c a_{1}, c a_{2}, \ldots, c a_{k}\right)$;

- denote by $(\mathbf{A}, \mathbf{B})$, or simply $\mathbf{A B}$, the concatenation: $\mathbf{A B}=\left(a_{1}, \ldots, a_{k}, b_{1}, \ldots, b_{k^{\prime}}\right)$;

- denote by $(c, \mathbf{A})$ the concatenation of $(c)$ and $\mathbf{A}:(c, \mathbf{A})=\left(c, a_{1}, a_{2}, \ldots, a_{k}\right)$

- denote by $\mathbf{A}^{i-}$ the suffix of $\mathbf{A}$ starting at position $i$ : $\mathbf{A}^{i-}=\left(a_{i}, a_{i+1}, \ldots, a_{k}\right)$.

The following is the round elimination lemma for $\mathrm{LPM}_{m, n}^{\Sigma}$ that plays a central role in proving the lower bound. The lemma is generalized from a simpler round elimination lemma in [10] to adapt to the non-uniform amount of information communicated in each round.

Lemma 19 (round elimination lemma for $\mathrm{LPM}_{m, n}^{\Sigma}$ ) Let $m, n, p, q$ be positive integers such that $2 p|m, q| n$, and $n \leq|\Sigma|$. Let $0<\epsilon, \delta<1$ and $\mathbf{A}, \mathbf{B} \in \mathbb{R}_{\geq 0}^{k}$. There is a universal constant $C>0$ such that the followings hold. Assume that $k \geq 1$ and $\frac{2 a_{1}}{p} \geq C$. If $\mathrm{LPM}_{m, n}^{\Sigma}$ has a private-coin $\langle\mathbf{A}, \mathbf{B}, 2 k\rangle^{A}$-protocol with error probability $\epsilon$, then $\mathrm{LPM}_{m / 2 p, n / q}^{\Sigma}$ has a private-coin $\left\langle\mathbf{A}^{\prime}, \mathbf{B}^{\prime}, 2 k-2\right\rangle^{A_{-}}$ protocol with error probability $\epsilon^{\prime}$, where

$$
\mathbf{A}^{\prime}=\left(1+\frac{2 a_{1}}{\delta p a_{2}}\right) \mathbf{A}^{2-}, \quad \mathbf{B}^{\prime}=\mathbf{B}^{2-}, \quad \text { and } \epsilon^{\prime}=\epsilon+2 \delta+\sqrt{b_{1} 2^{\frac{2 a_{1}}{\delta p}} / q} .
$$

The rest of Section 4.2 is dedicated to the proof of this lemma. The proof is almost identical to the one in [10], except for the part dealing with non-uniform message sizes. We include the proof here for the completeness of the paper.

We only need to show that the following two propositions.

Part I. Assume that $k \geq 1$ and $2 a_{1} / p \geq C$. If $\operatorname{LPM}_{m, n}^{\Sigma}$ has a private-coin $\langle\mathbf{A}, \mathbf{B}, 2 k\rangle^{A}$-protocol with error probability $\epsilon$, then $\operatorname{LPM}_{m / p, n}^{\Sigma}$ has a private-coin $\left\langle\mathbf{A}^{\prime}, \mathbf{B}^{\prime}, 2 k-1\right\rangle^{B}$-protocol with error probability $\epsilon+2 \delta$, where

$$
\mathbf{A}^{\prime}=\left(1+\frac{2 a_{1}}{\delta p a_{2}}\right) \mathbf{A}^{2-} \quad \text { and } \quad \mathbf{B}^{\prime}=\left(b_{1} 2^{\frac{2 a_{1}}{\delta p}}, \mathbf{B}^{2-}\right)
$$

Part II. Assume that $n \leq|\Sigma|$. If $\operatorname{LPM}_{m, n}^{\Sigma}$ has a private-coin $\left\langle\mathbf{A},\left(b_{0}, \mathbf{B}\right), 2 k+1\right\rangle^{B}$-protocol with error probability $\epsilon$, then $\operatorname{LPM}_{m-1, n / q}^{\Sigma}$ has a private-coin $\langle\mathbf{A}, \mathbf{B}, 2 k\rangle^{A}$-protocol with error probability $\epsilon+\sqrt{b_{0} / q}$. 
The round elimination lemma (Lemma 19) follows by combining these two propositions together, and weakening the resulting statement from $m / p-1$ to $m / 2 p$. The proofs of these two propositions will follow the same routine as in [10], with a generalization to deal with non-uniform message sizes.

The following is a typical proposition in the context of round elimination of communication protocols. Here we prove a version which is suitable for our setting.

Lemma 20 (message switching lemma) Let $P$ be a deterministic $\langle\mathbf{A}, \mathbf{B}, 2 k\rangle^{A}$-protocol with $k \geq 1$. Then there exists a deterministic $\left\langle\mathbf{A}^{\prime}, \mathbf{B}^{\prime}, 2 k-1\right\rangle^{B}$-protocol, where $\mathbf{A}^{\prime}=\left(1+a_{1} / a_{2}\right) \mathbf{A}^{2-}$ and $\mathbf{B}^{\prime}=\left(b_{1} 2^{a_{1}}, \mathbf{B}^{2-}\right)$, that computes the exact same problem as $P$.

Proof: There are at most $2^{a_{1}}$ different messages that Alice may send as the first message. Bob starts the new protocol by sending his at most $2^{a_{1}}$ different responses as in $P$. If $k=1$, the new protocol stops after this. Otherwise, let Alice's first message be the concatenation of her first two messages in $P$. And then the protocol continues just as in $P$. This increases the sizes of Alice's messages (in fact only her first message in the new protocol) by a factor of at most $\left(1+a_{1} / a_{2}\right)$.

We need to define some concepts for the information complexity of communications. Let $P$ be a communication protocol and $\mathcal{D}$ a joint distribution on the possible inputs to Alice and Bob. Let $\operatorname{err}(P, \mathcal{D})$ denote the probability of $P$ being error under input distribution $\mathcal{D}$. Let $\mathcal{D}_{A}$ denote the marginal distribution of $\mathcal{D}$ on Alice's inputs and $\mathcal{D}_{B}$ the marginal distribution on Bob's inputs.

Definition 21 (information cost) The information cost of a private-coin protocol $P$ with respect to input distribution $\mathcal{D}$, denoted $\operatorname{icost}(P, \mathcal{D})$, is defined to be the mutual information $I(X$ : $\operatorname{msg}(P, X)$ ), where $X$ is a random input drawn from $\mathcal{D}_{A}$ (if Alice starts $P$ ) or $\mathcal{D}_{B}$ (if Bob starts $P)$, and $\operatorname{msg}(P, x)$ denotes the first message in protocol $P$ if the sender's input is $x$.

The next two generic lemmas hold for general communication protocols with non-uniform sizes of messages, which apply to our setting.

Lemma 22 (uninformative message lemma [22]) Let $P$ be a private-coin $\langle\mathbf{A}, \mathbf{B}, 2 k\rangle^{A}$-protocol for a communication problem $\rho$. Then for any input distribution $\mathcal{D}$, there is a deterministic $\left\langle\mathbf{A}^{2-}, \mathbf{B}, 2 k-1\right\rangle^{B}$-protocol $P^{\prime}$ for $\rho$ such that $\operatorname{err}\left(P^{\prime}, \mathcal{D}\right) \leq \operatorname{err}(P, \mathcal{D})+\sqrt{\operatorname{icost}(P, \mathcal{D})}$.

Lemma 23 (message compression lemma [10]) Let $P$ be a private-coin $\langle\mathbf{A}, \mathbf{B}, 2 k\rangle^{A}$-protocol for a communication problem $\rho$. Then for any input distribution $\mathcal{D}$ and any $a>0$, there is a deterministic $\left\langle\left(a, \mathbf{A}^{2-}\right), \mathbf{B}, 2 k\right\rangle^{A}$-protocol $P^{\prime}$ for $\rho$ such that $\operatorname{err}\left(P^{\prime}, \mathcal{D}\right) \leq \operatorname{err}(P, \mathcal{D})+(2 \cdot \operatorname{icost}(P, \mathcal{D})+$ $C) / a$, where $C>0$ is a universal constant.

Now we are ready to show the two propositions that support the round elimination lemma. This is done by going through the same proof in [10] with a different parameterization.

Proof of Part I. Assume that $\operatorname{LPM}_{m, n}^{\Sigma}$ has private-coin $\langle\mathbf{A}, \mathbf{B}, 2 k\rangle^{A}$-protocol with error probability $\epsilon$ for a $k \geq 1$. We then construct a private-coin $\left\langle\mathbf{A}^{\prime}, \mathbf{B}^{\prime}, 2 k-1\right\rangle^{B}$-protocol with error probability $\epsilon+2 \delta$ for $\mathrm{LPM}_{m / p, n}^{\Sigma}$ where $\mathbf{A}^{\prime}$ and $\mathbf{B}^{\prime}$ are given in (7), when $2 a_{1} / p \geq C$ for a universal constant $C>0$.

Let $S \triangleq \Sigma^{m / p}$. By Yao's min-max principle, it suffices to give a deterministic protocol for $\mathrm{LPM}_{m / p, n}^{\Sigma}$ with the same message lengths and distributional error on any input distribution $\mathcal{D}$ on $S \times S^{n}$. Fix an input distribution $\mathcal{D}$ over $S \times S^{n}$. Define the following distributions: 
$\mathcal{I}$ : Let $\mathcal{I}$ defenote the distribution over $[p] \times S^{*}$ obtained as follows: choose $i \in[p]$ uniformly at random and draw $\sigma \in S^{i-1}$ from $\mathcal{D}_{A}^{i-1}$. Recall that $\mathcal{D}_{A}$ denote the marginal distribution on Alice's inputs.

$\mathcal{D}_{i, \sigma}$ : Let $s$ be some arbitrarily fixed element in $S$. For each pair $(i, \sigma)$ of $\mathcal{I}$ we define a distribution $\mathcal{D}_{i, \sigma}$ on $S^{p} \times\left(S^{p}\right)^{n}$ as follows: draw a sample $(x, y)$ from $\mathcal{D}$, independently draw $p-i$ strings $X_{i+1}, \cdots, X_{p}$ from $\mathcal{D}_{A}$, then output $\left(\sigma x X_{i+1} \cdots X_{p}, \sigma y s^{p-i}\right)$. Note that $y$ is a set of string, and the $\sigma y s^{p-i}$ denote the set of strings $\left\{\sigma \tau s^{p-i}: \tau \in y\right\}$.

$\widetilde{\mathcal{D}}$ : Finally, let $\widetilde{\mathcal{D}}$ be the distribution on $S^{p} \times\left(S^{p}\right)^{n}$ obtained by drawing a $(i, \sigma)$ from $\mathcal{I}$ and outputting a sample from $\mathcal{D}_{i, \sigma}$.

By the easy direction of Yao's min-max principle, there is a deterministic $\langle\mathbf{A}, \mathbf{B}, 2 k\rangle^{A}$-protocol $P$ for $\operatorname{LPM}_{m, n}^{\Sigma}$ with distributional error at most $\epsilon$ under distribution $\widetilde{\mathcal{D}}$. By definition,

$$
\mathbf{E}_{i, \sigma}\left[\operatorname{err}\left(P, \mathcal{D}_{i, \sigma}\right)\right]=\operatorname{err}(P, \widetilde{\mathcal{D}}) \leq \epsilon,
$$

where the expectation is taken over $(i, \sigma)$ which is sampled from $\mathcal{I}$.

Let $X=X_{1} X_{2} \cdots X_{p}$ be distributed according to $\widetilde{\mathcal{D}}_{A}=\mathcal{D}_{A}^{p}$. Then by definition,

$$
\begin{aligned}
\operatorname{icost}(P, \widetilde{\mathcal{D}}) & =\mathrm{I}(X: \operatorname{msg}(P, X)) \\
& =\sum_{i \in[p]} \mathrm{I}\left(X_{i}: \operatorname{msg}(P, X) \mid X_{1} \cdots X_{i-1}\right) \\
& =\sum_{i \in[p]} \mathbf{E}_{\sigma}\left[\mathrm{I}\left(X_{i}: \operatorname{msg}(P, X) \mid X_{1} \cdots X_{i-1}=\sigma\right)\right] \\
& =p \cdot \mathbf{E}_{i, \sigma}\left[\mathrm{I}\left(X_{i}: \operatorname{msg}(P, X) \mid X_{1} \cdots X_{i-1}=\sigma\right)\right] .
\end{aligned}
$$

Note that $\operatorname{icost}(P, \widetilde{\mathcal{D}}) \leq a_{1}$, since Alice's first message is of length $a_{1}$. Hence

$$
\mathbf{E}_{i, \sigma}\left[\mathrm{I}\left(X_{i}: \operatorname{msg}(P, X) \mid X_{1} \cdots X_{i-1}=\sigma\right)\right] \leq \frac{a_{1}}{p}
$$

Due to the linearity of expectation,

$$
\begin{aligned}
& \mathbf{E}_{i, \sigma}\left[\operatorname{err}\left(P, \mathcal{D}_{i, \sigma}\right)+\frac{2 \cdot \mathrm{I}\left(X_{i}: \operatorname{msg}(P, X) \mid X_{1} \cdots X_{i-1}=\sigma\right)}{2 a_{1} /(\delta p)}\right] \\
= & \mathbf{E}_{i, \sigma}\left[\operatorname{err}\left(P, \mathcal{D}_{i, \sigma}\right)\right]+\frac{2 \mathbf{E}_{i, \sigma}\left[\mathrm{I}\left(X_{i}: \operatorname{msg}(P, X) \mid X_{1} \cdots X_{i-1}=\sigma\right)\right]}{2 a_{1} /(\delta p)} \\
\leq & \epsilon+\frac{2 a_{1} / p}{2 a_{1} /(\delta p)}=\epsilon+\delta .
\end{aligned}
$$

By the averaging principle, there is an integer $i \in[p]$ and a string $\sigma \in S^{i-1}$ such that

$$
\operatorname{err}\left(P, \mathcal{D}_{i, \sigma}\right)+\frac{2 \cdot \mathrm{I}\left(X_{i}: \operatorname{msg}(P, X) \mid X_{1} \cdots X_{i-1}=\sigma\right)}{2 a_{1} /(\delta p)} \leq \epsilon+\delta .
$$

Fix the pair $(i, \sigma)$ to satisfy above. We can now construct a private-coin protocol $Q^{\prime \prime}$ for $\operatorname{LPM}_{m / p, n}^{\Sigma}$ which uses $P$ as a black box. It works as follows: given an input $(x, y) \in S \times S^{n}$, Alice constructs 
a string $\tilde{x} \triangleq \sigma x X_{i+1} \cdots X_{p}$ where the $X_{j}$ 's are random strings drawn independently from $\mathcal{D}_{A}$ using her private-coins, and Bob constructs the set of strings $\tilde{y} \triangleq \sigma y s^{p-i}$. They then run protocol $P$ on input $(\tilde{x}, \tilde{y})$ and output the $i$-th block of the output of $P$. Note that $(\tilde{x}, \tilde{y})$ is distributed according to $\mathcal{D}_{i, \sigma}$ if $(x, y)$ is distributed according to $\mathcal{D}$. Clearly, due to the definition of LPM, $Q^{\prime \prime}$ works as $P$ works. Therefore,

$$
\operatorname{err}\left(Q^{\prime \prime}, \mathcal{D}\right) \leq \operatorname{err}\left(P, \mathcal{D}_{i, \sigma}\right)
$$

Moreover,

$$
\operatorname{icost}\left(Q^{\prime \prime}, \mathcal{D}\right)=\mathrm{I}\left(X_{i}: \operatorname{msg}(P, X) \mid X_{1} \cdots X_{i-1}=\sigma\right) .
$$

Applying the message compression lemma (Lemma23) to $Q^{\prime \prime}$, we have a deterministic $\left\langle\left(2 a_{1} /(\delta p), \mathbf{A}^{2-}\right), \mathbf{B}, 2 k\right\rangle^{A_{-}}$ protocol $Q^{\prime}$ for $\operatorname{LPM}_{m / p, n}^{\Sigma}$, whose error on distribution $\mathcal{D}$ is bounded as

$$
\begin{aligned}
\operatorname{err}\left(Q^{\prime}, \mathcal{D}\right) & \leq \operatorname{err}\left(Q^{\prime \prime}, \mathcal{D}\right)+\left(2 \cdot \operatorname{icost}\left(Q^{\prime \prime}, \mathcal{D}\right)+C\right) /\left(2 a_{1} /(\delta p)\right) \\
& \leq \epsilon+\delta+\delta \frac{C}{2 a_{1} / p}
\end{aligned}
$$

Recall that we assume $2 a_{1} / p \geq C$. The above error is bounded by $\epsilon+2 \delta$.

Applying the message switching lemma (Lemma 20) to $Q^{\prime}$, we have a deterministic $\left\langle\mathbf{A}^{\prime}, \mathbf{B}^{\prime}, 2 k-\right.$ $1\rangle^{B}$-protocol $Q$ with error probability $\epsilon+2 \delta$ for $\mathrm{LPM}_{m / p, n}^{\Sigma}$ where $\mathbf{A}^{\prime}$ and $\mathbf{B}^{\prime}$ are given in (7). Applying this to every joint distribution $\mathcal{D}$ over the inputs with Yao's min-max lemma, we prove the first proposition supporting the round elimination lemma.

Proof of Part II. Assume that $n \leq|\Sigma|$ and $\operatorname{LPM}_{m, n}^{\Sigma}$ has a private-coin $\left\langle\mathbf{A},\left(b_{0}, \mathbf{B}\right), 2 k+1\right\rangle^{B}$ protocol with error probability $\epsilon$. Let $S \triangleq \Sigma^{m-1}$. For an arbitrary input distribution $\mathcal{D}$ on $S \times S^{n / q}$, we show the existence of a deterministic $\langle\mathbf{A}, \mathbf{B}, 2 k\rangle^{A}$-protocol for $\operatorname{LPM}_{m-1, n / q}^{\Sigma}$ with error $\epsilon+\sqrt{b_{0} / q}$ on distribution $\mathcal{D}$. By Yao's min-max principle, this is sufficient.

Since $q \mid n$ implies that $q \leq n \leq|\Sigma|$, we can fix $q$ distinct strings $s_{1}, \cdots, s_{q} \in \Sigma$. We now define two distributions based on $\mathcal{D}$.

$\mathcal{D}_{i}$ : For each $i \in[q]$, let $\mathcal{D}_{i}$ be the distribution on $\Sigma S \times(\Sigma S)^{n}$ obtained as follows: independently draw $q$ samples $\left(x_{1}, y_{1}\right), \cdots,\left(x_{q}, y_{q}\right)$ from $\mathcal{D}$, and output $\left(s_{i} x_{i}, s_{1} y_{1} \cup \cdots \cup s_{q} y_{q}\right)$.

$\widetilde{\mathcal{D}}$ : We also contruct a distribution $\widetilde{\mathcal{D}}$ on $\Sigma S \times(\Sigma S)^{n}$ as follows: choose $i \in[q]$ uniformly at random, and output a sample from $\mathcal{D}_{i}$.

By the easy direction of Yao's min-max principle there is a deterministic $\left\langle\mathbf{A},\left(b_{0}, \mathbf{B}\right), 2 k+1\right\rangle^{B}$ protocol $P$ for $\operatorname{LPM}_{m, n}^{\Sigma}$ with error at most $\epsilon$ on input distribution $\widetilde{\mathcal{D}}$. By definition,

$$
\mathbf{E}_{i}\left[\operatorname{err}\left(P, \mathcal{D}_{i}\right)\right]=\operatorname{err}(P, \widetilde{\mathcal{D}}) \leq \epsilon
$$

Let $Y=Y_{1} Y_{2} \cdots Y_{q}$ be distributed according to $\widetilde{\mathcal{D}}_{B}$, where $\widetilde{\mathcal{D}}_{B}$ is the marginal distribution of $\widetilde{\mathcal{D}}$ on Bob's inputs. We have

$$
\begin{aligned}
\operatorname{icost}(P, \widetilde{\mathcal{D}}) & =\mathrm{I}(Y: \operatorname{msg}(P, Y)) \\
& \geq \sum_{i \in[q]} \mathrm{I}\left(Y_{i}: \operatorname{msg}(P, Y)\right), \quad\left(Y_{i}^{\prime} \text { 's are independent }\right) \\
& =q \cdot \mathbf{E}_{i}\left[\mathrm{I}\left(Y_{i}: \operatorname{msg}(P, Y)\right)\right] .
\end{aligned}
$$


Note that $\operatorname{icost}(P, \widetilde{\mathcal{D}}) \leq b_{0}$ since Bob's first message is of length $b_{0}$. Hence

$$
\mathbf{E}_{i}\left[\mathrm{I}\left(Y_{i}: \operatorname{msg}(P, Y)\right)\right] \leq \frac{b_{0}}{q} .
$$

Due to the linearity of expectation,

$$
\begin{aligned}
& \mathbf{E}_{i}\left[\operatorname{err}\left(P, \mathcal{D}_{i}\right)+\mathrm{I}\left(Y_{i}: \operatorname{msg}(P, Y)\right)\right] \\
= & \mathbf{E}_{i}\left[\operatorname{err}\left(P, \mathcal{D}_{i}\right)\right]+\mathbf{E}_{i}\left[\mathrm{I}\left(Y_{i}: \operatorname{msg}(P, Y)\right)\right] \\
\leq & \epsilon+b_{0} / q
\end{aligned}
$$

By the averaging principle and the concavity of the square root function, there is an $i \in[q]$ such that

$$
\operatorname{err}\left(P, \mathcal{D}_{i}\right)+\sqrt{\mathrm{I}\left(Y_{i}: \operatorname{msg}(P, Y)\right)} \leq \epsilon+\sqrt{b_{0} / q}
$$

Fix the $i$ as above. We can now define a private-coin protocol $Q^{\prime}$ for $\operatorname{LPM}_{m-1, n / q}^{\Sigma}$ which uses $P$ as a black box. It works as follows: given an input $(x, y) \in S \times S^{n / q}$, Alice constructs a string $\tilde{x} \triangleq s_{i} x$ and Bob constructs the set of strings $\tilde{y} \triangleq s_{1} y_{1} \cup \cdots \cup s_{i-1} y_{i-1} \cup s_{i} y \cup s_{i+1} y_{i+1} \cup \cdots \cup s_{q} y_{q}$ where the $y_{j}$ 's are random sets of strings drawn independently from $\mathcal{D}_{B}$ using his private-coins. They then run protocol $P$ on input $(\tilde{x}, \tilde{y})$ and output the second block of the output of $P$. Note that the $(\tilde{x}, \tilde{y})$ is distributed according to $\mathcal{D}_{i}$ if $(x, y)$ is distributed according to $\mathcal{D}$. Clearly, due to the definition of LPM, $Q^{\prime}$ works as $P$ works. Therefore,

$$
\operatorname{err}\left(Q^{\prime}, \mathcal{D}\right) \leq \operatorname{err}\left(P, \mathcal{D}_{i}\right)
$$

Moreover,

$$
\operatorname{icost}\left(Q^{\prime}, \mathcal{D}\right)=\mathrm{I}\left(Y_{i}: \operatorname{msg}(P, Y)\right) .
$$

Applying the uninformative message lemma (Lemma 22) to $Q^{\prime}$, we have a deterministic $\langle\mathbf{A}, \mathbf{B}, 2 k\rangle^{A_{-}}$ protocol $Q$ for $\operatorname{LPM}_{m-1, n / q}^{\Sigma}$ with error at most $\epsilon+\sqrt{b_{0} / q}$ on distribution $\mathcal{D}$. Applying this to every joint distribution $\mathcal{D}$ over the inputs with Yao's min-max lemma, we prove the second proposition supporting the round elimination lemma.

\subsection{Proof of the lower bound}

We now prove the communication lower bound for $\operatorname{LPM}_{m, n}^{\Sigma}$, by the round elimination tool we setup in previous sections.

Theorem 24 (communication lower bound for LPM) For any $c_{1}, c_{2}>0$, there exists a $c_{3}>$ 0 such that the followings hold. Let $n, d \geq 1$ be sufficiently large integers, and suppose that $d \leq$ $2^{\sqrt{\log n}}$ and $n \leq 2^{d^{0.99}}$. Let $\gamma \geq 3$ and $m=\left\lfloor(\log d)^{\eta \beta}\right\rfloor$, where $\eta$ and $\beta$ are as defined in (15). Let $\Sigma$ be a set of cardinality $\left\lceil 2^{d^{0.99}}\right\rceil$. Let $1 \leq k \leq \frac{\log \log d}{2 \log \log \log d}$ be an integer. Let $\mathbf{A}, \mathbf{B} \in \mathbb{R}_{>0}^{k}$ be in the form that $a_{i}=c_{1} t_{i} \log n$ and $b_{i}=t_{i} d^{c_{2}}$ for some $t_{i}>0$ for every $1 \leq i \leq k$. If $\mathrm{LPM}_{m, n}^{\Sigma}$ has $a$ private-coin $\langle\mathbf{A}, \mathbf{B}, 2 k\rangle^{A}$-protocol, then $t \triangleq \sum_{i=1}^{k} t_{i}>\frac{c_{3}}{k}\left(\log _{\gamma} d\right)^{1 / k}$.

Proof: Although $\mathbf{A}=\left(a_{1}, a_{2}, \ldots, a_{k}\right)$ and $\mathbf{B}=\left(b_{1}, b_{2}, \ldots, b_{k}\right)$, we additionally define

$$
a_{k+1} \triangleq a_{1}, \quad b_{k+1} \triangleq b_{1}, \quad \text { and } t_{k+1} \triangleq t_{1} .
$$


We set $\delta=\frac{1}{4 k}, p=\frac{1}{2} m^{1 / k}$, and further define

$$
c_{5} \triangleq \max _{1 \leq i \leq k} \frac{\log \left(b_{i} / \delta^{2}\right)}{\log n} \frac{m^{1 / k}}{k t_{i+1}} .
$$

By definitions of $\beta$ and $\eta$ in (5), we have

$$
\eta \beta=1-\frac{c_{4}+\log \log \gamma}{\log \log d}+\frac{c_{4} \log \log \gamma}{(\log \log d)^{2}},
$$

and

$$
m=(\log d)^{\eta \beta}=\frac{(\log d) 2^{c_{4} \log \log \gamma / \log \log d}}{2^{c_{4}} \log \gamma}=\Theta\left(\log _{\gamma} d\right) .
$$

Therefore, assuming that $d \leq 2^{\sqrt{\log n}}$ and $\gamma \geq 3$, we have

$$
\begin{aligned}
c_{5} & =\max _{i \in[k]} O\left(\frac{(\log d)^{1 / k}(\log k+\log d)}{k t_{i+1} \log n}\right) \\
& =O\left(\frac{(\log d)^{2}}{\log n}\right)=O(1),
\end{aligned}
$$

where the constant factor depends on $c_{2}$.

Furthermore, since $k \leq \frac{\log \log d}{2 \log \log \log d}$, it can be verified that

$$
m>k^{2 k}
$$

Now we define

$$
\xi \triangleq \frac{m^{1 / k}}{k}=\Theta\left(\frac{\left(\log _{\gamma} d\right)^{1 / k}}{k}\right) .
$$

We start our proof by assuming $\operatorname{LPM}_{m, n}^{\Sigma}$ has a private-coin $\langle\mathbf{A}, \mathbf{B}, 2 k\rangle^{A}$-protocol with error probability $1 / 8$ and

$$
t=c_{3} \xi=\xi /\left(c_{5}+16 c_{1} e^{16}\right),
$$

and derive an impossible result, which will prove that $t>c_{3} \xi$. For notational convenience, we ignore divisibility issues.

With the above assumption, we make the following claim.

Claim 25 For any non-negative integer $i \leq k, \operatorname{LPM}_{m_{i}, n_{i}}^{\Sigma}$ has a private-coin $\left\langle\mathbf{A}_{i}, \mathbf{B}_{i}, 2(k-i)\right\rangle^{A_{-}}$ protocol with error probability $\frac{1}{8}+3 i \delta$, where

$$
\begin{aligned}
m_{i} & =\frac{m a_{i+1}}{(2 p)^{i} a_{1}}, \\
n_{i} & =n^{1-\frac{1}{t} \sum_{j \leq i} t_{j+1}}, \\
\mathbf{A}_{i} & =\prod_{j=1}^{i}\left(1+\frac{2 a_{j}}{a_{j+1} \delta p}\right) \mathbf{A}^{(i+1)-}, \\
\mathbf{B}_{i} & =\mathbf{B}^{(i+1)-} .
\end{aligned}
$$


We prove this claim by induction on $i$. For $i=0$, the claim holds by our assumption. For induction hypothesis: assume the claim for an $i<k$. We then prove the claim for $i+1$.

We choose $p_{i+1}=\frac{a_{i+1}}{a_{i+2}} p$ and $q_{i+1}=n^{t_{i+2} / t}$. We claim that

$$
2 p_{i+1} \leq m_{i}=\frac{m a_{i+1}}{(2 p)^{i} a_{1}}
$$

When $i+1=k$, this is obviously true, because $\frac{2 p_{k}}{m_{k-1}}=\frac{2 a_{k} p}{a_{k+1}} \frac{a_{1}(2 p)^{k-1}}{m a_{k}}=1$ since $a_{k+1}=a_{1}$ and $2 p=m^{1 / k}$, thus $2 p_{k}=m_{k-1}$; and when $i<k-1$, we have $\frac{2 p_{i+1}}{m_{i}}=\frac{a_{1}(2 p)^{i}}{m a_{i+2}} \leq \frac{a_{1}}{a_{i+2} m^{1 / k}}$ because $2 p=m^{1 / k}$, and $\frac{a_{1}}{a_{i+2}} \leq t_{i} \leq t \leq m^{1 / k}$ (or otherwise $t>m^{1 / k}=\Omega\left(\left(\log _{\gamma} d\right)^{1 / k}\right)$ and there is nothing to prove). Therefore, $2 p_{i+1} \leq m_{i}$ holds for all $i$.

It is also obvious that

$$
q_{i+1} \leq n^{1-\frac{1}{t} \sum_{j=1}^{i} t_{j+1}} \leq|\Sigma|
$$

On the other hand, the quantity $\frac{2 a_{i, 1}}{p_{i+1}}$, where $a_{i, 1}=a_{i+1} \prod_{j=1}^{i}\left(1+\frac{2 a_{j}}{a_{j+1} \delta p}\right)$ is the first entry of $\mathbf{A}_{i}$, is bounded as below:

$$
\frac{2 a_{i+1}}{p_{i+1}} \prod_{j=1}^{i}\left(1+\frac{2 a_{j}}{a_{j+1} \delta p}\right) \geq \frac{2 a_{i+2}}{p} \geq \frac{2 c_{1} t_{i+2} \log n}{\frac{1}{2} m^{1 / k}}>\frac{2 c_{1} \log n}{(\log d)^{1 / k}}
$$

which is $\omega(1)$ for $d \leq 2^{\sqrt{\log n}}$. Therefore, it holds that $\frac{2 a_{i, 1}}{p_{i+1}}>C$ for the first entry $a_{i, 1}$ of $\mathbf{A}_{i}$, where $C$ is the universal constant in Lemma 19, Together with (10) and (11), the condition of the round elimination lemma (Lemma 19) is satisfied. We now apply the round elimination lemma to the protocol assumed by the induction hypothesis, to obtain a private-coin $\left\langle\mathbf{A}_{i+1}, \mathbf{B}_{i+1}, 2(k-i-1)\right\rangle^{A_{-}}$ protocol for $\operatorname{LPM}_{m_{i+1}, n_{i+1}}^{\Sigma}$ with error probability $\frac{1}{8}+3 \delta+2 \delta+\delta^{\prime}$, where

$$
\delta^{\prime}=\sqrt{\frac{b_{i+1}}{q_{i+1}}} \exp \left(\frac{a_{i+1} \ln 2}{\delta p_{i+1}} \prod_{j=1}^{i}\left(1+\frac{2 a_{j}}{a_{j+1} \delta p}\right)\right) .
$$

We then show that $\delta^{\prime} \leq \delta$. Note that this will finish our induction and prove Claim 25.

By (9), we have $\delta p / 2=\frac{1}{16} m^{1 / k} / k>\frac{k}{16}$, thus

$$
\begin{aligned}
\prod_{j=1}^{i}\left(1+\frac{2 a_{j}}{a_{j+1} \delta p}\right) & \leq \prod_{j=1}^{k}\left(1+\frac{2 a_{j}}{a_{j+1} \delta p}\right) \\
& \leq\left(1+\frac{2}{\delta p}\right)^{k} \\
& \leq \mathrm{e}^{16} .
\end{aligned}
$$

Therefore,

$$
\delta^{\prime} \leq \sqrt{\frac{b_{i+1}}{q_{i+1}}} 2^{\frac{e^{16} a_{i+1}}{\delta p_{i+1}}}=\sqrt{b_{i+1}} n^{8 e^{16} c_{1} t_{i+2} k / m^{1 / k}-t_{i+2} / 2 t} .
$$


Recall that $t=c_{3} \xi=\xi /\left(c_{5}+16 c_{1} e^{16}\right)$ and $c_{5}=\max _{1 \leq i \leq k} \frac{\log \left(b_{i} / \delta^{2}\right)}{\log n} \frac{m^{1 / k}}{k t_{i+1}}$. We have

$$
\begin{aligned}
& c_{5} \geq \frac{m^{1 / k} \log \left(b_{i+1} / \delta^{2}\right)}{k t_{i+2} \log (n)} \\
\Longleftrightarrow & 16 c_{1} e^{16}-\frac{m^{1 / k} \log \left(\delta^{2} / b_{i+1}\right)}{k t_{i+2} \log (n)} \leq 16 c_{1} e^{16}+c_{5}=\frac{1}{c_{3}} \\
\Longleftrightarrow & \left(16 c_{1} e^{16}-\frac{1}{c_{3}}\right) \frac{t_{i+2} k}{m^{1 / k}} \leq \frac{\log \left(\delta^{2} / b_{i+1}\right)}{\log n} \\
\Longleftrightarrow & \frac{16 e^{16} c_{1} t_{i+2} k}{m^{1 / k}}-\frac{t_{i+2}}{t} \leq \frac{\log \left(\delta^{2} / b_{i+1}\right)}{\log n} \\
\Longleftrightarrow & \delta^{\prime 2} \leq n^{16 e^{16} c_{1} t_{i+2} k / m^{1 / k}-t_{i+2} / t} b_{i+1} \leq \delta^{2} .
\end{aligned}
$$

And we prove that $\delta^{\prime} \leq \delta$. Claim 25 is proved.

Now let $i=k$ in Claim 25. We have a private-coin protocol without message exchange between Alice and Bob but solving $\operatorname{LPM}_{1,1}^{\Sigma}$ with error probability at most $\frac{1}{8}+\frac{3}{4}$. However, this is impossible due to the following claim.

Claim 26 Any private-coin protocol for $\mathrm{LPM}_{1,1}^{\Sigma}$ without message exchange can succeed with probability at most $1 /|\Sigma|$ in the worst case.

By Yao's min-max principle, it is sufficient to prove the lower bound for deterministic protocols on a uniform random inputs. Note that the only thing a deterministic Alice can do without communication is to pick a string in $\Sigma$ and output it, but this can only succeed with probability at most $1 /|\Sigma|$ for a random input.

Theorem 24 together with the translation from cell-probing scheme to communication protocol (Proposition 18) and the reduction from $\mathrm{LPM}_{m, n}^{\Sigma}$ to $\mathrm{ANNS}_{d, n}^{\gamma}$ (Lemma 14) prove the $k$-round cellprobe lower bound for $\mathrm{ANNS}_{d, n}^{\gamma}$ (Theorem 12), the main result of this section.

\section{References}

[1] M. Ajtai. A lower bound for finding predecessors in yao's cell probe model. Combinatorica, $8(3): 235-247,1988$.

[2] A. Andoni and P. Indyk. Near-optimal hashing algorithms for approximate nearest neighbor in high dimensions. In Foundations of Computer Science, 2006. FOCS'06. 47th Annual IEEE Symposium on, pages 459-468. IEEE, 2006.

[3] A. Andoni, P. Indyk, H. L. Nguyen, and I. Razenshteyn. Beyond locality-sensitive hashing. In Proceedings of the Twenty-Fifth Annual ACM-SIAM Symposium on Discrete Algorithms, pages 1018-1028. SIAM, 2014.

[4] A. Andoni and I. Razenshteyn. Optimal data-dependent hashing for approximate near neighbors. In Proceedings of the Forty-Seventh Annual ACM on Symposium on Theory of Computing, pages 793-801. ACM, 2015. 
[5] J. Aspnes, D. Eisenstat, and Y. Yin. Low-contention data structures. In Proceedings of the twenty-second annual ACM symposium on Parallelism in algorithms and architectures, pages 345-354. ACM, 2010.

[6] O. Barkol and Y. Rabani. Tighter bounds for nearest neighbor search and related problems in the cell probe model. In Proceedings of the thirty-second annual ACM symposium on Theory of computing, pages 388-396. ACM, 2000.

[7] A. Borodin, R. Ostrovsky, and Y. Rabani. Lower bounds for high dimensional nearest neighbor search and related problems. In Proceedings of the thirty-first annual ACM symposium on Theory of computing, pages 312-321. ACM, 1999.

[8] J. Brody and K. G. Larsen. Adapt or die: Polynomial lower bounds for non-adaptive dynamic data structures. Theory OF Computing, 11(19):471-489, 2015.

[9] A. Chakrabarti, B. Chazelle, B. Gum, and A. Lvov. A lower bound on the complexity of approximate nearest-neighbor searching on the hamming cube. In Discrete and Computational Geometry, pages 313-328. Springer, 2003.

[10] A. Chakrabarti and O. Regev. An optimal randomized cell probe lower bound for approximate nearest neighbor searching. SIAM Journal on Computing, 39(5):1919-1940, 2010.

[11] P. Indyk. Nearest neighbors in high-dimensional spaces. 2004.

[12] P. Indyk and R. Motwani. Approximate nearest neighbors: towards removing the curse of dimensionality. In Proceedings of the thirtieth annual ACM symposium on Theory of computing, pages 604-613. ACM, 1998.

[13] T. Jayram, S. Khot, R. Kumar, and Y. Rabani. Cell-probe lower bounds for the partial match problem. In Proceedings of the thirty-fifth annual ACM symposium on Theory of computing, pages 667-672. ACM, 2003.

[14] E. Kushilevitz and N. Nisan. Communication Complexity. Cambridge University Press, 2006.

[15] E. Kushilevitz, R. Ostrovsky, and Y. Rabani. Efficient search for approximate nearest neighbor in high dimensional spaces. SIAM Journal on Computing, 30(2):457-474, 2000.

[16] D. Liu. A strong lower bound for approximate nearest neighbor searching. Information Processing Letters, 92(1):23-29, 2004.

[17] P. B. Miltersen, N. Nisan, S. Safra, and A. Wigderson. On data structures and asymmetric communication complexity. In Proceedings of the twenty-seventh annual ACM symposium on Theory of computing, pages 103-111. ACM, 1995.

[18] I. Newman. Private vs. common random bits in communication complexity. Information processing letters, 39(2):67-71, 1991.

[19] R. Panigrahy, K. Talwar, and U. Wieder. A geometric approach to lower bounds for approximate near-neighbor search and partial match. In Foundations of Computer Science, 2008. FOCS'08. IEEE 49th Annual IEEE Symposium on, pages 414-423. IEEE, 2008. 
[20] R. Panigrahy, K. Talwar, and U. Wieder. Lower bounds on near neighbor search via metric expansion. In Foundations of Computer Science (FOCS), 2010 51st Annual IEEE Symposium on, pages 805-814. IEEE, 2010.

[21] M. Pătraşcu and M. Thorup. Higher lower bounds for near-neighbor and further rich problems. In Foundations of Computer Science, 2006. FOCS'06. 47th Annual IEEE Symposium on, pages 646-654. IEEE, 2006.

[22] P. Sen. Lower bounds for predecessor searching in the cell probe model. In Computational Complexity, 2003. Proceedings. 18th IEEE Annual Conference on, pages 73-83. IEEE, 2003.

[23] A. C.-C. Yao. Some complexity questions related to distributive computing (preliminary report). In Proceedings of the eleventh annual ACM symposium on Theory of computing, pages 209-213. ACM, 1979.

[24] A. C.-C. Yao. Should tables be sorted? Journal of the ACM (JACM), 28(3):615-628, 1981. 